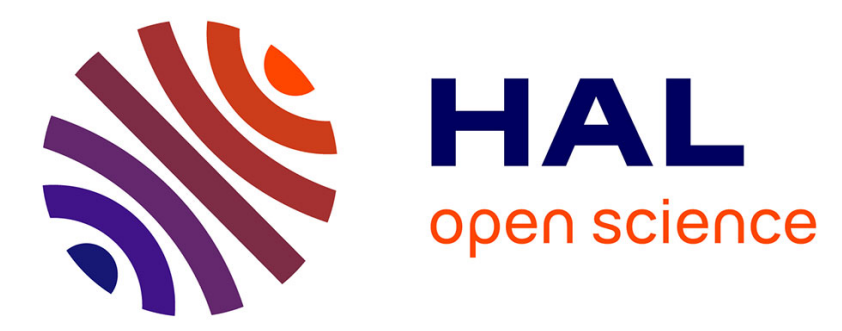

\title{
Computing the dynamics of biomembranes by combining conservative level set and adaptive finite element methods
}

\author{
Aymen Laadhari, Pierre Saramito, Chaouqi Misbah
}

\section{To cite this version:}

Aymen Laadhari, Pierre Saramito, Chaouqi Misbah. Computing the dynamics of biomembranes by combining conservative level set and adaptive finite element methods. Journal of Computational Physics, 2014, 263, pp.328-352. 10.1016/j.jcp.2013.12.032 · hal-00604145v4

\section{HAL Id: hal-00604145 \\ https://hal.science/hal-00604145v4}

Submitted on 7 Jan 2014

HAL is a multi-disciplinary open access archive for the deposit and dissemination of scientific research documents, whether they are published or not. The documents may come from teaching and research institutions in France or abroad, or from public or private research centers.
L'archive ouverte pluridisciplinaire HAL, est destinée au dépôt et à la diffusion de documents scientifiques de niveau recherche, publiés ou non, émanant des établissements d'enseignement et de recherche français ou étrangers, des laboratoires publics ou privés. 


\title{
Computing the dynamics of biomembranes by combining conservative level set and adaptive finite element methods
}

\author{
Aymen Laadhari ${ }^{\mathrm{a}, *}$, Pierre Saramito ${ }^{\mathrm{a}, * *}$, Chaouqi Misbah ${ }^{\mathrm{b}}$ \\ ${ }^{a}$ Lab. Jean Kuntzmann - CNRS and Université de Grenoble, F-38041 Grenoble, France \\ ${ }^{b}$ Lab. Interdisciplinaire de Physique, Université Joseph Fourier and CNRS, F-38041 \\ Grenoble France
}

\begin{abstract}
The numerical simulation of the deformation of vesicle membranes under simple shear external fluid flow is considered in this paper. A saddle-point approach is proposed for the imposition of the fluid incompressibility and the membrane inextensibility constraints, through Lagrange multipliers defined in the fluid and on the membrane respectively. Using a level set formulation, the problem is approximated by mixed finite elements combined with an automatic adaptive mesh procedure at the vicinity of the membrane boundary. Numerical experiments show that this combination of the saddle-point and adaptive mesh method enhances the robustness of the method. The effect of inertia on the stability of the vesicle in a shear flow is also investigated.
\end{abstract}

Keywords: level set method, mass conservation, adaptive finite element method, Helfrich energy, vesicle dynamics, fluid mechanics

\section{Introduction}

Phospholipid membranes are abundant in biology. They represent the major component of the cytoplasmic membrane of real cells. They are also present within the cell cytoplasm, e.g. the Golgi apparatus, a complex assembly of phospholipid layers which serve to form small vesicles for protein transport. Phospholipid membranes are also used in many industrial applications, as in

* Present address: Mathematics institute of computational science and engineering, École polytechnique fédérale de Lausanne, Lausanne, Switzerland

${ }^{* *}$ Corresponding author

Email addresses: aymen.laadhari@epfl.ch (Aymen Laadhari),

Pierre.Saramito@imag.fr (Pierre Saramito), chaouqi.misbah@ujf-grenoble.fr (Chaouqi Misbah)

Preprint

January 7, 2014 
giant liposome emulsions for cosmetics. A simple closed membrane of pure phospholipid suspended in an aqueous solution, also called a suspension of vesicles, constitute an attractive model system in order to describe mechanical and viscoelastic behaviors of many cells, like red blood cells. They are also considered as promising drug carriers for a delivery at specific sites in the organisms. This explains the increasing interest for biological membranes from various communities ranging from biology $[48,55]$ to applied mathematics $[6,29,52]$. This contribution is concerned with a certain aspect of mathematical modeling of vesicles, and more generally of phospholipid membranes.

Vesicles are formed by amphiphilic molecules self-assembling in water to build bilayers, in a certain range of concentration and temperature. At room, as well as at the physiological temperature, the membrane is a two dimensional incompressible fluid. Due to incompressibility, the main mode of deformation of a vesicle is bending. A basic ingredient for biomembranes is thus bending energy. Canham [13] and Helfrich [25, 43] introduced the following expression of the bending energy:

$$
\frac{k}{2} \int_{\Gamma}\left(H-H_{0}\right)^{2} \mathrm{~d} s+\frac{k_{g}}{2} \int_{\Gamma} K \mathrm{~d} s,
$$

where $H=H_{1}+H_{2}$ is the total curvature of the membrane surface, with $H_{1}$ and $H_{2}$ are the principle curvatures and $K=H_{1} H_{2}$ is the Gauss curvature. The membrane surface is denoted by $\Gamma$ while $\Omega$ represents the volume inside the vesicle, such that $\Gamma=\partial \Omega$. The integrals are performed along the membrane surface where $\mathrm{d} s$ denotes a surface area, while, in this paper, $\mathrm{d} x$ will represent a volume element. The constants $k$ and $k_{g}$ have the dimension of an energy and represent the bending modulus and the Gaussian curvature modulus, respectively. Here, $H_{0}$ denotes the spontaneous curvature that describes the asymmetry of the membrane. In this paper, $H_{0}=0$, since $H_{0}$ is relevant only for threedimensional problems (see appendix AppendixA) and we restrict ourself to the two-dimensional case in this paper. Finally, from the Gauss-Bonnet theorem, the second term of the Canham-Helfrich energy (1) is a topological invariant. Since topological changes are not considered in this paper, this second term is omitted.

Vesicles can be more or less inflated: the deflation could be due to osmotic effects, depending on additives in the solution. It could also be due to thermal effects: the thermal expansion of phospholipids is greater than those of the water inside the membrane, and thus, the area $A_{0}$ of the vesicle increases more rapidly than its volume $V_{0}$. In three dimension, the reduced volume, denoted by $\gamma$, measures the deflation:

$$
\left.\left.\gamma=\frac{3 V_{0}}{4 \pi} \times\left(\frac{4 \pi}{A_{0}}\right)^{3 / 2} \in\right] 0,1\right] .
$$

Thus, $\gamma$ compares the vesicle volume $V_{0}$ with the volume of a sphere having the area equal to $A_{0}: \gamma$ is a dimensionless number, that equals to 1 when the vesicle is a sphere and is lower than 1 otherwise. For instance, for the human red blood cell $\gamma \approx 0.64$. By varying $\gamma$, the shape that minimizes the energy of curvature 
can vary from an ellipsoid stretched to a biconcave shape, towards forms varied as that of the Peanut. In the two-dimensional case, $V_{0}$ and $A_{0}$ denotes the area and the perimeter respectively. The reduced area $\gamma$ compares the area of the vesicle with the area of a circle having the same perimeter as the vesicle. The reduced area is expressed in the two-dimensional case by:

$$
\gamma=\frac{V_{0}}{\pi} \times\left(\frac{2 \pi}{A_{0}}\right)^{2} .
$$

For a circle, the reduced area equals to 1 . The membrane could be considered as inextensible. In order to satisfy this inextensibility constraint, two approaches are commonly available. The first one use the penalty approach, together with a penalty parameter (see e.g. $[15,18]$ ): the inextensibility constraint then is not exactly satisfied and the approximate solution depends upon the penalty parameter. Recently, Kim and al. [32] proposed a penalty immersed boundary method to simulate the dynamics of inextensible vesicle. A virtual force is introduced in order to take into account the inextensibility constraint. The second solution introduces a Lagrange multiplier, that is interpreted as the surface tension of the membrane, and the inextensibility constraints is exactly satisfied. In the present work, the second solution was selected, since it avoids the dependence of the solution upon the penalty parameter. This formulation based on Lagrange multipliers is of common use for incompressible fluid flow applications (see e.g. [51]).

Furthermore, for general interface fluid flow problems, there are two main classes of numerical methods usually used: the class of Lagrangian methods based on an explicit interface parameterization and discretization while the class of Eulerian methods uses an implicit function. The popular phase field and level set methods fall into this second class.

For Lagrangian methods, the interface, which represents the biological membrane, is discretized by a set of points which are moved with a speed depending on the studied problem. For the computation of static vesicle shapes, the classical finite element method has been extensively used for surface reconstruction problems [4]. In [14, 47] the authors proposed a semi-implicit variational formulation (see also [28] for another semi-implicit approach in the context of capillary problems). In [40], an augmented Lagrangian algorithm was introduced in order to enforce the volume and area conservation while computing the static shape of a vesicle. In [9], the volume and area conservation constraints was enforced at the discrete level to machine precision. For the dynamics of vesicles in interaction with a fluid, the mesh following the interface may regenerate at each time step, while the boundary conditions between inside and outside volume of the interface could be directly imposed on this explicit boundary. The older method used for vesicle fluid applications falls into this category: the boundary element method transforms all viscous volume terms into surface integrals through a Green kernel and only a surface mesh of the interface is required [45] (see also $[46,8]$ ). Nevertheless, inertia terms are not reducible to boundary integrals and, despite some recent improvements, this approach suffers from some 
limitations. A more recent approach involves both two meshes: the first is localized on the interface and the second is a volume mesh. When the volume mesh is compatible with the interface discretization, the classical finite element method could be used [12]. A commonly used variant fixes the volume mesh one time for all and expresses interface integrals on a discrete moving surface mesh: this is the so called penalty immersed boundary method [32, 34].

The Eulerian methods are characterized by the use of a meshing strategy that is independent of the movements of the interface: this approach allows the use of fixed and fully structured volume mesh, and the surface mesh isn't anymore required to discretize the moving interface. Very complex shapes, with strong variations of the curvature and possible topological changes becomes possible. In the case of a diffuse interface, as for fluid mixtures, the interface is represented by a smooth transition zone. Indeed, at least at the molecular scale, there is a small zone of mixture between species. From a numerical point of view, the diffuse interface notion could be interpreted as a way of regularization of a sharp interface, together with a regularization parameter, associated to the interface width: this is the phase field method, introduced by Allen and Cahn [2], and applied recently to vesicles [19] and their fluid interactions [7, 17]. Moreover, the level set method [42] is an Eulerian approach that is able to catch sharp interfaces, where a transport equation is used to move the level set function and to describe the interface motion. This method is widely used to model vesicle dynamics [16, 41, 49, 50]. Nevertheless, both phase field and level set methods suffer a lack of precision when dealing with the volume and area conservation constraints. The aim of this paper is to present a new level set method that exactly solve these constraints at the discrete level at machine precision: it extends to the vesicle dynamics a previous work on level set methods for the advection equation [37].

We focus our attention on describing the dynamics of a single suspended vesicle in a linear shear gradient of a plane flow. Vesicles in shear flow in the limit of the vanishing Reynolds number (also called the Stokes limit) have been the subject of extensive studies [6]. Few works deals with the effect of fluid inertia on the dynamics of the vesicle: let us mention the pioneers works of [39] and $[49,50]$ that both observe a variation of inclination the angle of the vesicle under a shear flow when the Reynolds number increases. In the present work, non-zero Reynolds numbers are considered and the effect of inertia are more deeply investigated. This situation is of practical interest for red blood cells applications.

The outline of the paper is as follows. A saddle-point approach allows us to characterize the solution in a weak formulation, which is discretized using mixed finite elements in section 2. In Section 3 we focus on the numerical method. We present our level set method formulation for the vesicle dynamics and show the finite element discretization as well the advection mass preservation improvement. Section 4 is devoted to show numerical results illustrating the vesicle membrane in the tumbling and the tank-treading regimes. Finally, the effect of the inertia terms is explorated and we show that, beyond a critical value of the Reynolds number, the vesicle passes from a tumbling to a tank-treading regime. 


\section{Problem statement}

\subsection{Notations and preliminary results}

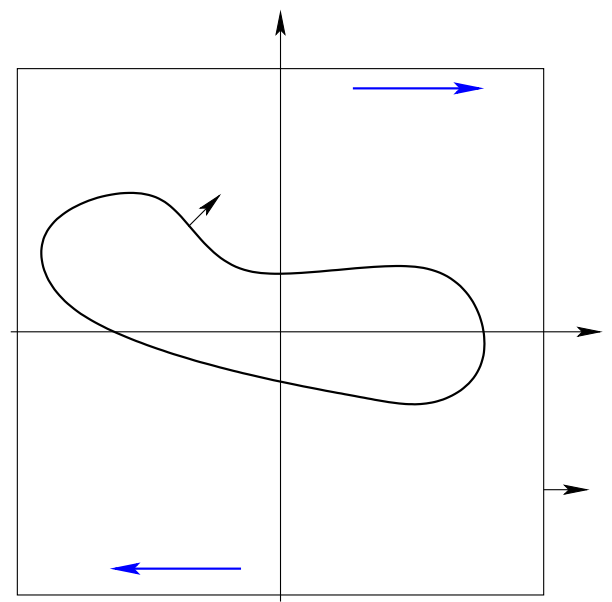

Figure 1: Notations for the vesicle interacting with a surrounding shear flow.

Let $\Lambda=]-L, L{ }^{d}$ be the bounded region containing the vesicle and the surrounding fluid, where $L>0$ is the half domain width. Numerical computations are performed in this paper with $d=2$, while the mathematical formulation could be extended to $d=3$ with few modifications. Let $T>0$ : for any $t \in] 0, T[$, the membrane $\Gamma(t) \subset \Lambda$ is the closed surface defined by:

$$
\Gamma(t)=\{(t, x) \in] 0, T[\times \Lambda ; \quad \phi(t, x)=0\},
$$

where $\phi$ is the level set function. By convention, the vesicle $\Omega(t) \subset \Lambda$ is the region where $\phi(t,$.$) is negative and we have \Gamma(t)=\partial \Omega(t)$.

Let $\mathbf{u}$ denote the velocity of the fluid in $\Lambda$. The membrane $\Gamma(t)$ is transported at the same velocity, and thus, the level set function satisfies:

$$
\left.\frac{D \phi}{D t}=\frac{\partial \phi}{\partial t}+\mathbf{u} \cdot \nabla \phi=0 \text { in }\right] 0, T[\times \Lambda,
$$

where $D / D t$ denotes the material derivative. The previous transport equation is completed by suitable boundary and initial conditions:

$$
\begin{aligned}
\phi & \left.=\phi_{b} \text { on }\right] 0, T\left[\times \Sigma_{-}\right. \\
\phi(0) & =\phi_{0} \text { in } \Lambda
\end{aligned}
$$

where

$$
\Sigma_{-}=\{x \in \partial \Lambda ; \mathbf{u} . \boldsymbol{\nu}(x)<0\}
$$


is the upstream boundary and $\boldsymbol{\nu}$ denotes the outward unit normal vector to the boundary $\partial \Lambda$. We remark that the solution of equation (4a) is affected by the upstream boundary condition (4b) on $\Sigma_{-}$; however it is not affected by the boundary condition imposed downstream. Concerning the initial condition (4c), the function $\phi_{0}$ denotes the signed distance between $x$ and the given initial shape membrane $\partial \Omega(0)$ :

$$
\phi_{0}(x)= \begin{cases}\inf \{|y-x| ; y \in \partial \Omega(0)\} & \text { when } x \notin \Omega(0), \\ \inf \{-|y-x| ; y \in \partial \Omega(0)\} & \text { otherwise. }\end{cases}
$$

Let $\mathbf{n}$ denote the unit outward normal vector to the shape $\Omega$ (see Fig. 1). Let $f$ be any scalar function and $\mathbf{v}$ be any vector field. The surface gradient, the surface divergence and the Laplace-Beltrami operator are respectively expressed by:

$$
\begin{aligned}
\nabla_{s} f & =(I-\mathbf{n} \otimes \mathbf{n}) \nabla f=\nabla f-(\mathbf{n} \cdot \nabla f) \mathbf{n} \\
\operatorname{div}_{s} \mathbf{v} & =(I-\mathbf{n} \otimes \mathbf{n}): \nabla \mathbf{v}=\operatorname{div} \mathbf{v}-((\nabla \mathbf{v}) \cdot \mathbf{n}) \cdot \mathbf{n}, \\
\Delta_{s} f & =\operatorname{div}_{s}\left(\nabla_{s} f\right) .
\end{aligned}
$$

Here, $\otimes$ denotes the tensorial product of two vectors and the semicolon : is the two times contracted product between tensors.

The mean and the Gauss curvatures can be expressed in terms of the normal $\mathbf{n}$ (see [36]):

$$
\begin{aligned}
H & =\nabla_{s} \cdot \mathbf{n}=\nabla \cdot \mathbf{n} \\
2 K & =H^{2}-\nabla \mathbf{n}: \nabla \mathbf{n}^{T} .
\end{aligned}
$$

\subsection{The dimensional problem}

The problem can be written as: 
find $\phi, \mathbf{u}, p$ and $\lambda$ such that

$$
\begin{aligned}
\frac{\partial \phi}{\partial t}+\mathbf{u} \cdot \nabla \phi & =0 \text { in }] 0, T[\times \Lambda \\
\rho\left(\frac{\partial \mathbf{u}}{\partial t}+\mathbf{u} \cdot \nabla \mathbf{u}\right)-\operatorname{div}(2 \eta D(\mathbf{u}))+\nabla p & =0 \text { in }] 0, T[\times(\Lambda \backslash \partial \Omega) \\
\operatorname{div} \mathbf{u} & =0 \text { in }] 0, T[\times \Lambda \\
\operatorname{div}_{s} \mathbf{u} & =0 \text { on }] 0, T[\times \partial \Omega \\
{[\mathbf{u}] } & =0 \text { on }] 0, T[\times \partial \Omega \\
-k\left\{\Delta_{s} H+H\left(\frac{H^{2}}{2}-2 K\right)\right\} \mathbf{n} & \\
+H \lambda \mathbf{n}-\nabla_{s} \lambda+[2 \eta D(\mathbf{u})-p I] . \mathbf{n} & =0 \text { on }] 0, T[\times \partial \Omega \\
\phi & \left.=\phi_{b} \text { on }\right] 0, T\left[\times \Sigma_{-}\right. \\
\mathbf{u} & \left.=\mathbf{u}_{b} \text { on }\right] 0, T\left[\times \Sigma_{D}\right. \\
(2 \eta D(\mathbf{u})-p I) . \nu & =0 \text { on }] 0, T\left[\times \Sigma_{N}\right. \\
\phi(0) & =\phi_{0} \text { in } \Lambda \\
\mathbf{u}(0) & =\mathbf{u}_{0} \text { in } \Lambda
\end{aligned}
$$

Equation (7a) expresses that the interface is transported by the fluid, and it is completed by the suitable boundary and initial conditions $(7 \mathrm{~g})$ and $(7 \mathrm{j})$ presented in the previous section, where the upstream boundary $\Sigma_{-}(5)$ depends on the flow velocity $\mathbf{u}$. Notice that the conservation of momentum $(7 \mathrm{~b})$ is written in $\Lambda \backslash \partial \Omega$, i.e. in $\Omega$ and its complementary $\Lambda \backslash \bar{\Omega}$. Here, $D(\mathbf{u})=\left(\nabla \mathbf{u}+(\nabla \mathbf{u})^{T}\right) / 2$ is the symmetric part of the gradient of velocity tensor. The viscosity $\eta$ is not constant over $\Lambda$ : it takes a constant value $\eta_{0}$ outside the vesicle $\Omega(t)$ and a different constant value $\eta_{1}$ inside the vesicle.

The unknown velocity field must satisfy two constraints: the fluid mass conservation (7c) and the membrane inextensibility (7d). The mass conservation reduces to the divergence-free condition $\operatorname{div} \mathbf{u}=0$ since the density, denoted by $\rho$, is supposed to be constant. Conversely, the membrane inextensibility is written locally as $\operatorname{div}_{s} \mathbf{u}=0$.

On $\partial \Omega$, [.] denotes the jump of a quantity across $\partial \Omega$ in the normal direction $\mathbf{n}$. Equation (7e) expresses the continuity of the velocity across the interface. The jump term in (7f) expresses the balance with membrane strengths. Indeed, the first normal term comes from the Canham-Helfrich bending energy (1) and is not an obvious computation (see $[36,43]$ ), since $\partial \Omega(t)$ and $H$ depend implicitly upon $\mathbf{u}$ : it requires some advanced shape optimization tools. This bending energy being a purely geometrical quantity, it cannot give rise to a tangential strength: any tangential movement of points on a surface is only modifying their positions without affecting the shape of the surface and its curvature energy. The second and third terms in (7f) involves the Lagrange multiplier $\lambda$ (the surface tension), and is defined on the membrane $\partial \Omega(t)$. The second term is normal and it is similar to the strengths of capillarities engendered by the surface 
tension when modeling the dynamics of drops. The term $\nabla_{s} \lambda$ is tangential and its action is known as the Marangoni effect.

The unknown level set $\phi$ and velocity field $\mathbf{u}$ satisfy some boundary and initial conditions.

The boundary $\left.\Sigma_{D}=\right]-L, L^{d-1} \times\{-L, L\}$ is associated to the Dirichlet boundary condition $\mathbf{u}_{b}(t, x)$, expressed for a shear flow by:

$$
\mathbf{u}_{b}(t, x)=\left\{\begin{aligned}
V & \text { when } x_{d}=L \\
-V & \text { when } x_{d}=-L
\end{aligned}\right.
$$

for all $(t, x) \in] 0, T\left[\times \Sigma_{D}\right.$. Here, $V$ denotes the given shear velocity at the box boundary (see Fig. 1). Conversely, $\left.\Sigma_{N}=\{-L, L\}^{d-1} \times\right]-L, L[$ is associated to a Neumann-type boundary condition.

\subsection{Dimensionless problem}

The characteristic length $R_{0}$ of the vesicle is chosen equal to the radius of a sphere having the same surface as the vesicle $\partial \Omega$ in the three dimensional case. In the two-dimensional case, it represents the radius of a circle having the same perimeter as $\partial \Omega$. The characteristic velocity $U=V R_{0} / L$ is chosen to be equal to the shear velocity at a distance $R_{0}$ from the center. The characteristic viscosity $\eta_{0}$ is chosen as the viscosity of the exterior fluid.

The following four dimensionless numbers are introduced:

$$
R e=\frac{\rho R_{0} U}{\eta_{0}}, \quad C a=\frac{\eta_{0} R_{0}^{2} U}{k}, \quad \alpha=\frac{R_{0}}{L} \text { and } \beta=\frac{\eta_{1}}{\eta_{0}} .
$$

The Reynolds number $R e$, as usual, expresses the ratio of inertia effects to the viscous ones. The capillarity number $C a$ compares the strength of the imposed flow, $\eta_{0} U / R_{0}$, to the bending resistance of the membrane $k / R_{0}^{3}$. The dimensionless number $\alpha$ represents the confinement of the vesicle in the shear flow and $\beta$ is the viscosity ratio. The initial shape $\partial \Omega(0)$ is also characterized by a fifth dimensionless number: $\gamma$, the reduced area, already introduced in Eq. (2).

In the rest of the paper, only dimensionless quantities are used and, since there is no ambiguity, they are still denoted with the same notations as the original quantities. The dimensionless version of the boundary condition for velocity is:

$$
\mathbf{u}_{b}(t, x)=\left\{\begin{aligned}
1 / \alpha & \text { when } x_{d}=\alpha \\
-1 / \alpha & \text { when } x_{d}=-\alpha
\end{aligned}\right.
$$

A dimensionless viscosity function is also defined:

$$
\eta_{*}(t, x)= \begin{cases}\beta & \text { when } x \in \Omega(t) \\ 1 & \text { otherwise }\end{cases}
$$


Then, the dimensionless problem is written with respect to the dimensionless variables and numbers. While equations in the dimensionless problem are still similar to those in (7), only equations (7b), (7f) and (7i) lead to the following ones

$$
\begin{aligned}
& R e\left(\frac{\partial \mathbf{u}}{\partial t}+\mathbf{u} \cdot \nabla \mathbf{u}\right)-\operatorname{div}\left(2 \eta_{*} D(\mathbf{u})\right)+\nabla p=0 \text { in }] 0, T[\times(\Lambda \backslash \partial \Omega) \\
&-\frac{1}{C a}\left\{\Delta_{s} H+H\left(\frac{H^{2}}{2}-2 K\right)\right\} \mathbf{n} \\
&\left.+H \lambda \mathbf{n}-\nabla_{s} \lambda+\left[2 \eta_{*} D(\mathbf{u})-p I\right] . \mathbf{n}=0 \text { on }\right] 0, T[\times \partial \Omega \\
&\left(2 \eta^{*} D(\mathbf{u})-p I\right) \cdot \boldsymbol{\nu}=0 \text { on }] 0, T\left[\times \Sigma_{N}\right.
\end{aligned}
$$

where the normal $\mathbf{n}=\nabla \phi /|\nabla \phi|$ and the curvature $H=\operatorname{div}_{s} \mathbf{n}$ are expressed in term of the level set function.

\subsection{Variational formulation}

Let us introduce the following space of admissible velocities:

$$
\mathbb{V}\left(\mathbf{u}_{b}\right)=\left\{\mathbf{v} \in\left(H^{1}(\Lambda)\right)^{d} ; \mathbf{v}=\mathbf{u}_{b} \text { on } \Sigma_{D}\right\}
$$

Equation (8a) is multiplied by a test function $\mathbf{v} \in \mathbb{V}(0)$ and then integrated by parts on $\Omega$ and $\Lambda \backslash \bar{\Omega}$ separately. Then, terms are merged and we get:

$$
\begin{array}{r}
\int_{\Lambda} R e \frac{D \mathbf{u}}{D t} \cdot \mathbf{v} \mathrm{d} x-\int_{\Lambda} \operatorname{div}\left(2 \eta^{*} D(\mathbf{u})-p I\right) \cdot \mathbf{v} \mathrm{d} x+\int_{\partial \Lambda}\left\{\left(2 \eta^{*} D(\mathbf{u})-p I\right) \cdot \boldsymbol{\nu}\right\} \cdot \mathbf{v} \mathrm{d} s \\
+\int_{\partial \Omega(t)}\left\{\left[2 \eta^{*} D(\mathbf{u})-p I\right] \cdot \mathbf{n}\right\} \cdot \mathbf{v} \mathrm{d} s-\int_{\partial \Omega(t)} \nabla_{s} \lambda \cdot \mathbf{v} \mathrm{d} s+\int_{\partial \Omega(t)} \lambda H \mathbf{n} \cdot \mathbf{v} \mathrm{d} s \\
=\int_{\partial \Omega(t)} \mathbf{f} \cdot \mathbf{v} \mathrm{d} s
\end{array}
$$

where $I$ is the identity tensor and div is the divergence of a symmetric tensor, defined as the divergence of its row or column vectors. We have introduced the material time derivative $\frac{D \mathbf{u}}{D t}=\partial_{t} \mathbf{u}+\mathbf{u} . \nabla \mathbf{u}$. The strength $\mathbf{f}$ appears in the right-hand-side of the previous equation and it describes the minimization of the Canham-Helfrich energy; it is given by:

$$
\mathbf{f}=\frac{1}{C a}\left\{\Delta_{s} H+H\left(\frac{H^{2}}{2}-2 K\right)\right\} \mathbf{n} .
$$

However, the Gauss curvature writes $K=0$ in the two-dimensional case and the force (9) reduces to: $\mathbf{f}=\mathrm{Ca}^{-1}\left(\Delta_{s} H+H^{3} / 2\right) \mathbf{n}$. We assume to have enough regularity for the interface $\partial \Omega(t)$, e.g. $C^{4}(\Omega)$, such that integrals involving $\mathbf{f}$ could be well defined. 
In order to deal with the integrals over $\partial \Omega(t)$, a generalization of the Green formula over the closed surface $\partial \Omega(t)$ is used (see e.g. [36]):

$$
\int_{\partial \Omega} \nabla_{s} \mu \cdot \mathbf{v} \mathrm{d} s+\int_{\partial \Omega} \mu \operatorname{div}_{s} \mathbf{v} \mathrm{d} s=\int_{\partial \Omega} \mu \mathbf{v} \cdot \mathbf{n} H \mathrm{~d} s, \quad \forall \mu \in H^{\frac{1}{2}}(\partial \Omega), \quad \forall \mathbf{v} \in H^{1}(\Lambda) .
$$

The equations (10) leads to the following variational formulation:

$$
\begin{aligned}
\text { find } \mathbf{u} \in C^{0}(] 0, T\left[, L^{2}(\Lambda)^{d}\right) \cap L^{2}(] 0, T\left[, \mathbb{V}\left(\mathbf{u}_{b}\right)\right), p \in L^{2}(] 0, T\left[, L_{0}^{2}(\Omega)\right) \\
\text { and } \lambda \in L^{2}(] 0, T\left[, H^{\frac{1}{2}}(\partial \Omega)\right) \text { such that } \\
\qquad \begin{aligned}
\int_{\Lambda} R e \frac{D \mathbf{u}}{D t} \cdot \mathbf{v} \mathrm{d} x+\int_{\Lambda} 2 \eta^{*} D(\mathbf{u}): D(\mathbf{v}) \mathrm{d} x & \\
+\int_{\Lambda} p \operatorname{div} \mathbf{v} \mathrm{d} x+\int_{\partial \Omega(t)} \lambda \operatorname{div}_{s} \mathbf{v} \mathrm{d} s & \left.=\int_{\partial \Omega(t)} \mathbf{f . v} \mathrm{d} s, \quad \forall \mathbf{v} \in \mathbb{V}(0), 11 \mathrm{a}\right) \\
\int_{\Lambda} q \operatorname{div} \mathbf{u} \mathrm{d} x & =0, \quad \forall q \in L^{2}(\Lambda), \\
\int_{\partial \Omega(t)} \mu \operatorname{div}_{s} \cdot \mathbf{u} \mathrm{d} s & =0, \quad \forall \mu \in H^{\frac{1}{2}}(\partial \Omega(t))
\end{aligned}
\end{aligned}
$$

where the initial condition is $\mathbf{u}(0)=\mathbf{u}_{0}$. Concerning the coupling with the level set problem, we recall that the interface $\partial \Omega(t)$ is given by (3), and it is described by using $\phi$, which is solution of the transport problem (4a)-(4c) involving the volicity vector $\mathbf{u}$. Moreover, we remark that the stationary problem can be expressed as a minimization one: this formulation is useful in order to understand the structure of the set of equations and the relations between the velocity field $\mathbf{u}$ and the two Lagrange multipliers $p$ and $\lambda$. Details on this formulation are provided in the Appendix (AppendixB).

\section{Numerical methods}

\subsection{Time discretization and the characteristic method}

The numerical computations are presented in the two-dimensional case, where the Gauss curvature $K$ is null. Nevertheless, the method extends to threedimensional case by including the computation of $K$ in the Canham-Helfrich force. Let $0=t^{0}<t^{1}<t^{2}<\cdots<t^{N}=T$ be a subdivision of the time interval $[0, T]$ with a constant time step $\Delta t=t^{n+1}-t^{n}, n=1,2, \cdots, N$. For $n=0, \phi^{0}=\phi_{0}$ represents the initial condition, and for any $n \geq 1$, the unknowns $\phi^{n}, \mathbf{u}^{n}, p^{n}$ and $\lambda^{n}$ at time step $n$ are computed by induction, using values at previous time steps. The time discretization is performed by using the method of characteristics: for any $t>0$ and $x \in \Lambda$, the characteristic curve $X(., x, ; t)$ passing at time $t$ through $x$ is defined by the following ordinary differential equation:

$$
\left\{\begin{aligned}
\frac{\partial X}{\partial t}(s, x ; t) & =\mathbf{u}(X(s, x ; t), t), \quad s \in] 0, T[ \\
X(t, x ; t) & =x .
\end{aligned}\right.
$$


For any function $\varphi(t, x)$, the total derivative $D \varphi / D t$ is expressed as:

$$
\frac{D \varphi}{D t}(t, x)=\left(\frac{\partial \varphi}{\partial t}+\mathbf{u} \cdot \nabla \varphi\right)(t, x)=\left.\frac{\partial}{\partial \tau}(\varphi(X(t, x ; \tau), \tau))\right|_{\tau=t}
$$

Following Pironneau [44], this derivative is approximated by a first-order backward Euler scheme:

$$
\frac{D \varphi}{D t}\left(t^{n}, x\right) \approx \frac{\varphi\left(t^{n}, x\right)-\varphi\left(t^{n-1}, X_{1}^{n-1}(x)\right)}{\Delta t}
$$

where $X_{1}^{n-1}(x)=x-\Delta t \mathbf{u}^{n-1}(x)$ denotes the first-order forward Euler approximation of $X\left(t^{n-1}, x ; t^{n}\right)$. The time-discretization of the transport equation (7a) leads to:

$$
\phi^{n}=\phi^{n-1} \circ X_{1}^{n-1} \text { in } \Lambda
$$

Then, the vesicle shape at step $n$ writes $\partial \Omega^{n}=\left\{x \in \Lambda ; \phi^{n}(x)=0\right\}$. The, the inner volume is given by $\Omega^{n}=\left\{x \in \Lambda ; \phi^{n}(x)<0\right\}$. The dimensionless viscosity is also computed explicitly:

$$
\eta_{*}^{n}= \begin{cases}\beta & \text { when } x \in \Omega^{n} \\ 1 & \text { otherwise }\end{cases}
$$

Moreover, the normal $\mathbf{n}^{n}$ and the mean curvature $H^{n}$ are updated, as well as the differential operators (6) on the surface $\partial \Omega^{n}$. Let $g \in \mathcal{C}^{3}([0, T])$ denotes an arbitrary function, a second order Taylor expansion writes:

$$
\frac{\mathrm{d} g}{\mathrm{~d} t}(t)=\frac{3 g(t)-4 g(t-\Delta t)+g(t-2 \Delta t)}{2 \Delta t}+\mathcal{O}\left(\Delta t^{2}\right) .
$$

Based on this approximation and following [51, chap. 5], the time discretization of the inertia term is performed by using a second order combined characteristic and finite difference discretization method. The second-order characteristics writes:

$$
\begin{aligned}
& \mathbf{u}^{*}=2 \mathbf{u}^{n-1}-\mathbf{u}^{n-2} \\
& X_{2}^{n-1}(x)=x-\Delta t \mathbf{u}^{*}(x) \text { a.e. } x \in \Lambda \text {, } \\
& X_{2}^{n-2}(x)=x-2 \Delta t \mathbf{u}^{*}(x) \text { a.e. } x \in \Lambda \text {, }
\end{aligned}
$$

where $\mathbf{u}^{*}$ represents a prediction by extrapolation of $\mathbf{u}$ at time $t_{n}$. Collecting the elements above, the problem becomes: 
find $\mathbf{u}^{n}, p^{n}$ and $\lambda^{n}$ such that

$$
\begin{aligned}
& \frac{R e}{2 \Delta t}\left(3 \mathbf{u}^{n}-4 \mathbf{u}^{n-1} o X_{2}^{n-1}+\mathbf{u}^{n-2} o X_{2}^{n-2}\right) \\
& -\operatorname{div}\left(2 \eta_{*}^{n} D\left(\mathbf{u}^{n}\right)\right)+\nabla p^{n}=0 \quad \text { in } \Lambda \backslash \partial \Omega^{n}(13 \mathrm{a}) \\
& \operatorname{div} \mathbf{u}^{n}=0 \text { in } \Lambda \\
& {\left[\mathbf{u}^{n}\right]=0 \text { on } \partial \Omega^{n} \text {, }} \\
& -\frac{1}{C a}\left(\Delta_{s}^{n} H^{n}+\frac{\left(H^{n}\right)^{3}}{2}\right) \mathbf{n}^{n}+H^{n} \lambda^{n} \mathbf{n}^{n}-\nabla_{s}^{n} \lambda^{n} \\
& +\left[2 \eta_{*}^{n} D\left(\mathbf{u}^{n}\right)-p^{n} \mathbf{I}\right] \cdot \mathbf{n}^{n}=0 \quad \text { on } \partial \Omega^{n}, \\
& \operatorname{div}_{s}^{n} \cdot \mathbf{u}^{n}=0 \text { on } \partial \Omega^{n} \text {, } \\
& \mathbf{u}^{n}=\mathbf{u}_{b} \text { on } \Sigma_{D} \text {. }
\end{aligned}
$$

The second order induction on $\left(\mathbf{u}^{n}\right)_{n \geq 0}$ is bootstrapped by using the initial condition: $\mathbf{u}^{-1}=\mathbf{u}^{0}=\mathbf{u}_{0}$, where $\mathbf{u}^{-1}$ stands here for a convenient notation. The previous scheme uses two main steps. The first step (12) is an explicit computation involving the characteristics. The second step (13) is a linear generalized Stokes sub-system that involves a constraint on the boundary of the vesicle together with the usual incompressibility constraint. We point out that this scheme transforms a strongly nonlinear shape optimization problem into a succession of explicit computations and linear subproblems. The next paragraph presents how such a linear subproblem is treated.

\subsection{The generalized Stokes subproblem}

\subsubsection{Formulation}

The Canham-Helfrich force (9) appears in the right-hand side of the generalized Stokes subproblem and is evaluated by using the updated values of $\mathbf{n}^{n}=\nabla \phi^{n} /\left|\nabla \phi^{n}\right|$ and $H^{n}=\operatorname{div}_{s} \mathbf{n}^{n}$ at time $t^{n}$. We introduce the weighted multi-linear forms:

$$
\begin{aligned}
m(\mathbf{u}, \mathbf{v}) & =\int_{\Lambda} \mathbf{u} \cdot \mathbf{v} \mathrm{d} x, \forall \mathbf{u}, \mathbf{v} \in\left(L^{2}(\Lambda)\right)^{2} \\
a^{n}(\mathbf{u}, \mathbf{v}) & =\int_{\Lambda} 2 \eta_{*}^{n} D(\mathbf{u}): D(\mathbf{v}) \mathrm{d} x, \forall \mathbf{u}, \mathbf{v} \in\left(H^{1}(\Lambda)\right)^{2} \\
b_{1}(\mathbf{v}, q) & =-\int_{\Lambda} q \operatorname{div} \mathbf{v} \mathrm{d} x, \forall q \in L^{2}(\Lambda), \forall \mathbf{v} \in H(\operatorname{div}, \Lambda), \\
b_{2}^{n}(\mathbf{v}, \mu) & =-\int_{\partial \Omega^{n}} \mu \operatorname{div}_{s}^{n} \mathbf{v} \mathrm{d} s, \forall \mu \in H^{\frac{1}{2}}\left(\partial \Omega^{n}\right), \forall \mathbf{v} \in H\left(\operatorname{div}_{s}, \partial \Omega^{n}\right),
\end{aligned}
$$

where $H(\operatorname{div}, \Lambda)=\left\{\mathbf{s} \in\left(L^{2}(\Lambda)\right)^{2} ; \operatorname{div} \mathbf{s} \in L^{2}(\Lambda)\right\}$ (see e.g. [11, 22]). The variational formulation of (13a)-(13f) writes: 
(S): find $\mathbf{u}^{n} \in \mathbb{V}\left(\mathbf{u}_{b}\right), p^{n} \in L^{2}(\Lambda)$ and $\lambda^{n} \in H^{\frac{1}{2}}\left(\partial \Omega^{n}\right)$ such that

$$
\begin{aligned}
& \frac{3 R e}{2 \Delta t} m\left(\mathbf{u}^{n}, \mathbf{v}\right)+a^{n}\left(\mathbf{u}^{n}, \mathbf{v}\right)+b_{1}\left(\mathbf{v}, p^{n}\right)+b_{2}^{n}\left(\mathbf{v}, \lambda^{n}\right) \\
& =m_{s}^{n}\left(\mathbf{f}^{n}, \mathbf{v}\right)+\frac{R e}{2 \Delta t} m\left(4 \mathbf{u}^{n-1} o X_{2}^{n-1}-\mathbf{u}^{n-2} o X_{2}^{n-2}, \mathbf{v}\right), \\
& b_{1}\left(\mathbf{u}^{n}, q\right)=0 \\
& b_{2}^{n}\left(\mathbf{u}^{n}, \mu\right)=0,
\end{aligned}
$$

for all $\mathbf{v} \in \mathbb{V}(0), q \in L^{2}(\Lambda)$ and $\mu \in H^{\frac{1}{2}}\left(\partial \Omega^{n}\right)$.

\subsubsection{The Canham-Helfrich force}

In this section, we are interested on the discretization of the Canham-Helfrich force (9). The force involves fourth order derivatives of the level set function and a direct discretization approach would require a highly regular finite element method, such as the Hermite one (see e.g. [10]) with $H^{2}$ and $C^{1}$ regularity. In order to use standard Lagrange finite element, with only $H^{1}$ and $C^{0}$ regularity, the fourth-order derivatives are treated here with a different approach, based on a duality argument.

Since $H^{n}=\operatorname{div} \mathbf{n}^{n}$ and $\mathbf{n}^{n}=\nabla \phi^{n} /\left|\nabla \phi^{n}\right|$, then $H^{n}$ involves the second order derivative of the level set function. Let us define the skeleton of $\Omega$ as the set of points that are equidistant to at least two distinct points of $\partial \Omega$ (see e.g. [3, p. 195]). In order to avoid division by $\left|\nabla \phi^{n}\right|$, that could vanish on the skeleton, two intermediate variables $\mathbf{r}^{n}=\nabla\left(\left|\nabla \phi^{n}\right|\right)$ and $G^{n}=H^{n}\left|\nabla \phi^{n}\right|$ are used. First, using a classical Green formula in $\Lambda, \mathbf{r}^{n}$ can be characterized as

$$
\mathbf{r}^{n} \in H_{0}(\operatorname{div}, \Lambda) \text { and } \int_{\Lambda} \mathbf{r}^{n} \cdot \mathbf{s} \mathrm{d} x=\int_{\Lambda}\left|\nabla \phi^{n}\right| \operatorname{div} \mathbf{s} \mathrm{d} x, \quad \forall \mathbf{s} \in H_{0}(\operatorname{div}, \Lambda),
$$

where $H_{0}(\operatorname{div}, \Lambda)=\{\mathbf{s} \in H(\operatorname{div}, \Lambda) ; \mathbf{s} . \boldsymbol{\nu}=0\}$. Next, let us turn to $G^{n}$. A simple development leads to:

$$
G^{n}\left|\nabla \phi^{n}\right|=-H^{n}\left|\nabla \phi^{n}\right|^{2}=-\operatorname{div}\left(\frac{\nabla \phi^{n}}{\left|\nabla \phi^{n}\right|}\right)\left|\nabla \phi^{n}\right|^{2}=\mathbf{r}^{n} \cdot \nabla \phi^{n}-\Delta \phi^{n}\left|\nabla \phi^{n}\right|
$$

The duality argument is used for the $\Delta \phi^{n}$ term at the right-hand side and $G^{n}$ is characterized by

$$
G^{n} \in H^{1}(\Lambda) \text { and } \int_{\Lambda} G^{n} \zeta\left|\nabla \phi^{n}\right| \mathrm{d} x=\int_{\Lambda}\left(\mathbf{r}^{n} \cdot \nabla \phi^{n}\right) \zeta \mathrm{d} x+\int_{\Lambda} \nabla \phi^{n} . \nabla \zeta\left|\nabla \phi^{n}\right| \mathrm{d} x, \forall \zeta \in H^{1}(\Lambda) .
$$

Finally, $H^{n}$ is defined as the restriction to $\partial \Omega^{n}$ of $G^{n} /\left|\nabla \phi^{n}\right|$. Notice that this quantity is well defined since $\left|\nabla \phi^{n}\right|$ does not vanish at the vicinity of $\partial \Omega^{n}$. Let us consider the following Green formula on the closed surface $\Gamma^{n}=\partial \Omega^{n}$ :

$$
\int_{\Gamma^{n}} \Delta_{s}^{n} \xi \zeta \mathrm{d} s+\int_{\Gamma^{n}} \nabla_{s}^{n} \xi \cdot \nabla_{s}^{n} \zeta \mathrm{d} s=0, \forall \xi, \zeta \in H^{1}\left(\Gamma^{n}\right) .
$$


Then $Y^{n}=-\Delta_{s} H^{n}$ can be computed in a weak sense:

$$
Y^{n} \in H^{1}\left(\partial \Omega^{n}\right) \text { and } \int_{\partial \Omega^{n}} Y^{n} \zeta \mathrm{d} s=\int_{\partial \Omega^{n}} \nabla_{s}^{n} H^{n} \cdot \nabla_{s}^{n} \zeta \mathrm{d} s, \forall \zeta \in H^{1}\left(\partial \Omega^{n}\right)
$$

Let us summarize the procedure, and we introduce the following additional bilinear forms:

$$
\begin{aligned}
m_{w}^{n}(\phi, \psi) & =\int_{\Lambda} \varphi \psi\left|\nabla \phi^{n}\right| \mathrm{d} x, \forall \varphi, \psi \in L^{2}(\Lambda), \\
a_{w}^{n}(\phi, \psi) & =\int_{\Lambda} \nabla \varphi \nabla \psi\left|\nabla \phi^{n}\right| \mathrm{d} x, \forall \varphi, \psi \in H^{1}(\Lambda), \\
m_{s}^{n}(\xi, \zeta) & =\int_{\partial \Omega^{n}} \xi \zeta \mathrm{d} s, \forall \xi, \zeta \in L^{2}\left(\partial \Omega^{n}\right), \\
c^{n}(\xi, \zeta) & =\int_{\partial \Omega^{n}} \nabla_{s}^{n} \xi \cdot \nabla_{s}^{n} \zeta \mathrm{d} s, \forall \xi, \zeta \in H^{1}\left(\partial \Omega^{n}\right) .
\end{aligned}
$$

Then, we compute successively:

$$
\begin{aligned}
\mathbf{r}^{n} \in H_{0}(\operatorname{div}, \Lambda) \text { such that } m\left(\mathbf{r}^{n}, \mathbf{s}\right) & =-b_{1}\left(\left|\nabla \phi^{n}\right|, \mathbf{s}^{n}\right), \forall \mathbf{s} \in H_{0}(\operatorname{div}, \Lambda), \\
G^{n} \in H^{1}(\Lambda) \text { such that } m_{w}^{n}\left(G^{n}, \psi\right) & =a_{w}^{n}\left(\phi^{n}, \psi\right)+m\left(\mathbf{r}^{n} \cdot \nabla \phi^{n}, \psi\right), \forall \psi \in H^{1}(\Lambda), \\
H^{n} & =\frac{G^{n}}{\left|\nabla \phi^{n}\right|} \text { on } \partial \Omega^{n}, \\
Y^{n} \in H^{1}\left(\partial \Omega^{n}\right) \text { such that } m_{s}^{n}\left(Y^{n}, \zeta\right) & =c^{n}\left(H^{n}, \zeta\right), \forall \zeta \in H^{1}\left(\partial \Omega^{n}\right), \\
\mathbf{n}^{n} & =\frac{\nabla \phi^{n}}{\left|\nabla \phi^{n}\right|} \text { on } \partial \Omega^{n}, \\
\mathbf{f}^{n} & =\frac{1}{C a}\left(-Y^{n}+\frac{\left(H^{n}\right)^{3}}{2}\right) \mathbf{n}^{n} \text { on } \partial \Omega^{n} .
\end{aligned}
$$

\subsubsection{Extension and regularization}

The previous variational formulation involves integrals over the moving surface $\partial \Omega^{n}$ : in order to avoid the explicit re-triangulation of the surface $\partial \Omega^{n}$ at each time step, integrals over $\partial \Omega^{n}$ are transformed into integrals over $\Lambda$. First, note that an integral over $\partial \Omega^{n}$ can be written as an integral over $\Lambda$ with the help of the level set function $\phi^{n}$ and the Dirac measure $\delta$ :

$$
\int_{\partial \Omega^{n}} \varphi \mathrm{d} s=\int_{\Lambda} \tilde{\varphi}\left|\nabla \phi^{n}\right| \delta\left(\phi^{n}\right) \mathrm{d} x
$$

where $\tilde{\varphi}$ is an extension to $\Lambda$ of any function $\varphi$ defined in $\partial \Omega^{n}$. Therefore, the normal vector $\mathbf{n}^{n}$, defined over $\partial \Omega^{n}$, is extended in the hole domain $\Lambda$ following $\nabla \phi^{n} /\left|\nabla \phi^{n}\right|$. Since there is no ambiguity, this extension of the normal is still denoted by $\mathbf{n}^{n}$. By the same way, we extend to $\Lambda$ the surface operators (6) and the Canham-Helfrich force (9); the previous notations are still conserved. Nevertheless, the explicit management of Dirac measures is not an easy task 
in finite element methods. Thus, the previous extension is combined together with a regularization procedure. Three sharp functions are here considered: the Heaviside function $\mathscr{H}\left(\phi^{n}\right)$, that acts as the indicator of $\Lambda \backslash \Omega^{n}$, the Dirac measure $\delta\left(\phi^{n}\right)$ that localizes the surface $\partial \Omega^{n}$, and the sign function $\operatorname{sgn}\left(\phi^{n}\right)$, that will be used in a forthcoming paragraph, for redistancing the level set function.

In order to avoid the triangulation of $\partial \Omega^{n}$, a banded region of width $2 \varepsilon$ is introduced, for some $\varepsilon>0$. The Heaviside $\mathscr{H}$, the Dirac $\delta$ and the sign functions are replaced respectively by $\mathscr{H}_{\varepsilon}, \delta_{\varepsilon}$ and $\operatorname{sgn}_{\varepsilon}$, defined for all $\phi \in \mathbb{R}$ by:

$$
\begin{aligned}
& \mathscr{H}_{\varepsilon}(\phi)= \begin{cases}0, & \text { when } \phi<-\varepsilon, \\
\frac{1}{2}\left(1+\frac{\phi}{\varepsilon}+\frac{\sin \left(\frac{\pi \phi}{\varepsilon}\right)}{\pi}\right), & \text { when }|\phi| \leq \varepsilon, \\
1, & \text { otherwise. }\end{cases} \\
& \delta_{\varepsilon}(\phi)=\frac{\mathrm{d} \mathscr{H}_{\varepsilon}}{\mathrm{d} \phi}(\phi)= \begin{cases}\frac{1}{2 \varepsilon}\left(1+\cos \left(\frac{\pi \phi}{\varepsilon}\right)\right), & \text { if }|\phi| \leq \varepsilon \\
0, & \text { otherwise. }\end{cases} \\
& \operatorname{sgn}_{\varepsilon}(\phi)=2 \mathscr{H}_{\varepsilon}(\phi)-1 .
\end{aligned}
$$

The sharp viscosity is also replaced by a smooth one: $\eta_{*, \varepsilon}^{n}=\beta+(1-\beta) \mathscr{H}_{\varepsilon}\left(\phi^{n}\right)$. The previous bilinear forms admits a regularized counterpart:

$$
\begin{aligned}
a_{\varepsilon}^{n}(\mathbf{u}, \mathbf{v}) & =\int_{\Lambda} 2 \eta_{*, \varepsilon}^{n} D(\mathbf{u}): D(\mathbf{v}) \mathrm{d} x, \forall \mathbf{u}, \mathbf{v} \in\left(H^{1}(\Lambda)\right)^{2}, \\
m_{s, \varepsilon}^{n}(\mathbf{u}, \mathbf{v}) & =\int_{\Lambda} \mathbf{u} . \mathbf{v}\left|\nabla \phi^{n}\right| \delta_{\varepsilon}\left(\phi^{n}\right) \mathrm{d} x, \forall \mathbf{u}, \mathbf{v} \in\left(L^{2}(\Lambda)\right)^{2}, \\
b_{2, \varepsilon}^{n}(\mathbf{v}, \mu) & =-\int_{\Lambda} \mu \operatorname{div}_{s}^{n} \mathbf{v}\left|\nabla \phi^{n}\right| \delta_{\varepsilon}\left(\phi^{n}\right) \mathrm{d} x, \forall \mu \in L^{2}(\Lambda), \forall \mathbf{v} \in\left(H^{1}(\Lambda)\right)^{2}, \\
c_{\varepsilon}^{n}(\xi, \zeta) & =\int_{\Lambda} \nabla_{s}^{n} \xi . \nabla_{s}^{n} \zeta\left|\nabla \phi^{n}\right| \delta_{\varepsilon}\left(\phi^{n}\right) \mathrm{d} x, \forall \xi, \zeta \in H^{1}(\Lambda) .
\end{aligned}
$$

The computation of the curvature $H^{n}$ is unchanged while the Canham-Helfrich force becomes: find $Y_{\varepsilon}^{n} \in H^{1}(\Lambda)$ such that

$$
m_{s, \varepsilon}\left(Y_{\varepsilon}^{n}, \zeta\right)=c_{\varepsilon}\left(H^{n}, \zeta\right), \forall \zeta \in H^{1}(\Lambda) .
$$

Then, compute the extension to $\Lambda$ of the force:

$$
\mathbf{f}_{\varepsilon}^{n}=\frac{1}{C a}\left(-Y_{\varepsilon}^{n}+\frac{\left(H^{n}\right)^{3}}{2}\right) \mathbf{n}^{n} \text { in } \Lambda .
$$

Problem (14) admits a regularized variant: 
$(S)_{\varepsilon}:$ find $\mathbf{u}_{\varepsilon}^{n} \in \mathbb{V}\left(\mathbf{u}_{b}\right), p_{\varepsilon}^{n} \in L^{2}(\Lambda)$ and $\lambda_{\varepsilon}^{n} \in L^{2}(\Lambda)$ such that

$$
\begin{aligned}
& \frac{3 R e}{2 \Delta t} m\left(\mathbf{u}_{\varepsilon}^{n}, \mathbf{v}\right)+a_{\varepsilon}^{n}\left(\mathbf{u}_{\varepsilon}^{n}, \mathbf{v}\right)+b_{1}\left(\mathbf{v}, p_{\varepsilon}^{n}\right)+b_{2, \varepsilon}^{n}\left(\mathbf{v}, \lambda_{\varepsilon}^{n}\right) \\
& =m_{s, \varepsilon}^{n}\left(\mathbf{f}_{\varepsilon}^{n}, \mathbf{v}\right)+\frac{R e}{2 \Delta t} m\left(4 \mathbf{u}_{\varepsilon}^{n} o X_{2}^{n}-\mathbf{u}_{\varepsilon}^{n-1} o X_{2}^{n-1}, \mathbf{v}\right), \\
& b_{1}\left(\mathbf{u}_{\varepsilon}^{n}, q\right)=0, \\
& b_{2, \varepsilon}^{n}\left(\mathbf{u}_{\varepsilon}^{n}, \mu\right)=0,
\end{aligned}
$$

for all $\mathbf{v} \in \mathbb{V}(0), q \in L^{2}(\Lambda)$ and $\mu \in L^{2}(\Lambda)$. We notice that the surface tension $\lambda_{\varepsilon}^{n}$ was extended to $\Lambda$. Concerning the regularization parameter $\varepsilon$, we choose it numerically proportional to the mean mesh size $h$.

\subsubsection{Finite element discretization}

The Taylor-Hood finite element approximation (see e.g. [11]) for the Stokes problem is considered here for the velocity-pressure approximation of the generalized Stokes problem. Let $\mathscr{T}_{h}$ a finite element triangulation of $\Lambda$, where $h>0$ stands for the largest element diameter [10]. The following finite dimensional spaces are introduced:

$$
\begin{aligned}
X_{h} & =\left\{q \in C^{0}(\bar{\Lambda}), q_{\mid K} \in \mathbb{P}_{1}, \forall K \in \mathscr{T}_{h}\right\}, \\
\mathbf{S}_{h} & =\left\{\mathbf{s} \in X_{h}^{2}, \mathbf{s . \nu}=0 \text { on } \partial \Lambda\right\} \\
\mathbb{X}_{h} & =\left\{\mathbf{u} \in\left(C^{0}(\bar{\Lambda})\right)^{2}, \mathbf{u}_{\mid K} \in\left(\mathbb{P}_{2}\right)^{d}, \forall K \in \mathscr{T}_{h}\right\}, \\
\mathbb{V}_{h}\left(\mathbf{u}_{b}\right) & =\mathbb{X}_{h} \cap \mathbb{V}\left(\mathbf{u}_{b}\right) .
\end{aligned}
$$

Let us assume that $\phi_{h}^{n} \in X_{h}$ is an approximation of $\phi^{n}$ at the $n$-th time step. The computation of the discrete Canham-Helfrich force is:

$$
\begin{aligned}
\mathbf{r}_{h}^{n} \in S_{h} \text { and } m\left(\mathbf{r}_{h}^{n}, \mathbf{s}\right) & =-b_{1}\left(\left|\nabla \phi_{h}^{n}\right|, \mathbf{s}^{n}\right), \forall \mathbf{s} \in S_{h}, \\
G_{h}^{n} \in X_{h} \text { and } m_{w}^{n}\left(G_{h}^{n}, \psi\right) & =a_{w}^{n}\left(\phi_{h}^{n}, \psi\right)+m\left(\mathbf{r}_{h}^{n} . \nabla \phi_{h}^{n}, \psi\right), \forall \psi \in X_{h}, \\
H_{h}^{n} & =\frac{G_{h}^{n}}{\left|\nabla \phi_{h}^{n}\right|} \text { in } \Lambda, \\
Y_{h}^{n} \in X_{h} \text { and } m_{s, \varepsilon}\left(Y_{h}^{n}, \zeta\right) & =c_{\varepsilon}\left(H_{h}^{n}, \zeta\right), \forall \zeta \in X_{h}, \\
\mathbf{n}_{h}^{n} & =\frac{\nabla \phi_{h}^{n}}{\left|\nabla \phi_{h}^{n}\right|} \text { in } \Lambda, \\
\mathbf{f}_{h}^{n} & =\frac{1}{C a}\left(-Y_{h}^{n}+\frac{\left(H_{h}^{n}\right)^{3}}{2}\right) \mathbf{n}_{h}^{n} \text { in } \Lambda .
\end{aligned}
$$

The discrete generalized Stokes problem is: 
$(S)_{h}:$ find $\mathbf{u}_{h}^{n} \in \mathbb{V}_{h}\left(\mathbf{u}_{b}\right), p_{h}^{n} \in X_{h}$ and $\lambda_{h}^{n} \in X_{h}$ such that

$$
\begin{aligned}
& \frac{3 R e}{2 \Delta t} m\left(\mathbf{u}_{h}^{n}, \mathbf{v}\right)+a_{\varepsilon}^{n}\left(\mathbf{u}_{h}^{n}, \mathbf{v}\right)+b_{1}\left(\mathbf{v}, p_{h}^{n}\right)+b_{2, \varepsilon}^{n}\left(\mathbf{v}, \lambda_{h}^{n}\right) \\
& =m_{s, \varepsilon}^{n}\left(\mathbf{f}_{h}^{n}, \mathbf{v}\right)+\frac{R e}{2 \Delta t} m\left(4 \mathbf{u}_{h}^{n-1} o X_{2}^{n-1}-\mathbf{u}_{h}^{n-2} o X_{2}^{n-2}, \mathbf{v}\right), \\
& b_{1}\left(\mathbf{u}_{h}^{n}, q\right)=0, \\
& b_{2, \varepsilon}^{n}\left(\mathbf{u}_{h}^{n}, \mu\right)=0,
\end{aligned}
$$

for all $\mathbf{v} \in \mathbb{V}_{h}(0), q \in X_{h}$ and $\mu \in X_{h}$. The previous finite-dimensional linear system involves the following matrix structure:

$$
\left(\begin{array}{ccc}
A & B_{1}^{T} & B_{2}^{T} \\
B_{1} & 0 & 0 \\
B_{2} & 0 & 0
\end{array}\right)
$$

Such systems have been extensively studied and various efficient strategies are known (see e.g. [21]). In the present paper, this system is solved efficiently by the preconditioned conjugate gradient algorithm, as implemented in the Rheolef $\mathrm{C}++$ library [51].

Summarising, the previous discrete algorithm involves a semi-implicit numerical scheme with respect to time : its convergence with respect to the time step choice will be investigated in the third part of this paper. However, the problem is highly non-linear and the Canham-Helfrich force includes a fourth derivative with respect to the level set function. As a matter of fact, restrictions on the time discretization step is needed for stability raisons. Consequently, this algorithm will be improved in the forthcoming section (3.4), and we present a fully-implicit scheme in time of type fix-point algorithm. This algorithm allows to use bigger time steps.

\subsection{The transport subproblem}

\subsubsection{Redistancing}

Due to the inextensibility of the vesicle membrane and the fluid incompressibility, the level set function $\phi$, initially chosen to be a signed distance, remains also, for any $t>0$, a signed distance among the advection step, as shown in appendix AppendixC. Nevertheless, after time and space discretization, we determined that the approximation $\phi_{h}$ is not a signed distance after the discrete counterpart of the advection step. As a consequence, an auxiliary problem called the redistance problem has to be solved in order to keep the function $\phi_{h}$ as a signed distance. The redistance step was detailed by the authors in a separate paper [37] and we recall here briefly the main idea. For all $t \in] 0, T[$, an advection problem depending on a pseudo-time $\tau$ is introduced and we shall find its stationary solution. Let $\tilde{\phi}(t,$.$) be the known level set function at time t$ that is 
no more a distance function. The redistancing problem writes:

$$
\left\{\begin{aligned}
\frac{\partial \psi}{\partial \tau}(\tau, x ; t)+\mathbf{v} \cdot \nabla \psi & =\operatorname{sgn}(\tilde{\phi}(t, x))+\lambda(\tau, x: t) g(\psi) & & \text { a.e. }(\tau, x) \in] 0,+\infty[\times \Lambda \\
\psi(0, x ; t) & =\tilde{\phi}(x, t) & & \text { a.e. } x \in \Lambda .
\end{aligned}\right.
$$

where the advection vector field is $\mathbf{v}=\operatorname{sgn}(\tilde{\phi}) \frac{\nabla \psi}{|\nabla \psi|}$ and $\operatorname{sgn}(\tilde{\phi})$ denotes the sign function and is equal to $0,-1,+1$ respectively on $\partial \Omega(t)$, inside $\Omega(t)$ and outside $\Omega(t)$. The redistancing problem (17) is affected by suitable Dirichlet boundary conditions that are applied on the upstream boundary $\Sigma_{-}$(5). However, as the advection vector $\mathbf{v}$ follows the outward normal vector to the vesicle surface, then the upstream domain $\Sigma_{-}$is an empty set, and solving the redistancing problem allows the level set to change values on $\partial \Lambda$. Besides, we notice that $\lambda(\tau, x ; t)$ is a Lagrange multiplier that enforces the constraint of constant volume locally at $x \in \Lambda$. The zero level set is not modified by the presence of $\lambda$ in the right hand side of (17). This multiplier was first introduced by Sussman and Fatemi [53] in a finite difference context and then extended in [37] in a finite element context.

Choosing $g(\psi)=\delta(\psi)|\nabla \psi|$, the Lagrange multiplier has an explicit average value $\lambda_{\mathcal{V}}$ over an arbitrary finite volume $\mathcal{V} \subset \Lambda$ :

$$
\lambda_{\mathcal{V}}(\tau ; t)= \begin{cases}\frac{\int_{\mathcal{V}} \delta(\psi)(\mathbf{v} \cdot \nabla \psi-\operatorname{sgn}(\tilde{\phi})) \psi x}{\int_{\mathcal{V}} \delta(\psi) g(\psi) \mathrm{d} x} & \text { when } \mathcal{V} \cap \partial \Omega(t) \neq \emptyset \\ 0 & \text { otherwise }\end{cases}
$$

The stationary solution satisfies $|\nabla \psi|=1$ almost everywhere in $\Lambda$, consequently $\psi(\infty, . ; t)$ is a signed distance and is taken as the new level set function $\phi(t,$. at time $t$. Let us notice that the solution $\psi$ of the redistance problem (17) preserves the position of $\partial \Omega(t)$ : for any $\tau>0$, the zero level set of $\psi(\tau, . ; t)$ is the same zero level set of $\phi(t,$.$) . As a result the volume meas (\Omega(t))$ is also preserved, this point has great importance for numerous applications. However, after discretization by finite difference or finite element methods, this property is satisfied only approximately. We introduce the redistance operator defined by $\phi(t,)=.\operatorname{redistance}(\tilde{\phi}(t,)$.$) .$

Let $\tilde{\phi}^{n}$ be the approximation of $\tilde{\phi}(t)$, at time $t^{n}$ and $\psi^{m}, \mathbf{v}^{m}$ be approximations of $\psi(\tau), \mathbf{v}(\tau)$ respectively at $\tau^{m}$. The time discretization is performed by using the method of characteristics and the total derivative $D \psi / D t$ is approximated by a first-order backward Euler scheme as previously. The redistance problem (17) is solved explicitly:

$$
\psi^{m+1}= \begin{cases}\psi^{m} & \text { when }\left|\tilde{\phi}^{n}\right|<\varepsilon \\ \psi^{m} \circ X_{\mathbf{v}_{\varepsilon}}^{m}+\Delta \tau \operatorname{sgn}_{\varepsilon}\left(\tilde{\phi}^{n}\right) & \text { otherwise }\end{cases}
$$

Here, the characteristics have subscripts $\mathbf{v}_{\varepsilon}$ in order to avoid confusion. Let $W_{h}$ be the space of piecewise constant functions on $\mathcal{T}_{h}$ and $\pi_{h}$ denotes the 
Lagrange interpolation in $Q_{h}$. Let $\psi_{h}^{0}=\tilde{\phi}_{h}^{n+1}$. At any step $m \geq 0$ of the redistance algorithm, suppose $\psi_{h}^{m} \in Q_{h}$ being known, and let $\mathbf{g}_{h}^{m} \in Q_{h}^{d}$ be the approximation of $\nabla \psi_{h}^{m} \in W_{h}^{d}$ defined by the following linear system:

$$
\int_{\Lambda} \mathbf{g}_{h}^{m} \cdot \boldsymbol{w}_{h} \mathrm{~d} x=\int_{\Lambda} \nabla \psi_{h}^{m} \cdot \boldsymbol{w}_{h} \mathrm{~d} x, \quad \forall \boldsymbol{w}_{h} \in Q_{h}^{d}
$$

A mass lumping procedure is used for this linear system: the integrals involved in the computation of the coefficients of the matrix associated to the $L^{2}$ scalar product are evaluated by using the trapeze quadrature formula. By this way, the matrix of the linear system is replaced by a diagonal one, and the computation of $\mathbf{g}_{h}^{m}$ becomes explicit. Then, let

$$
\mathbf{v}_{\varepsilon, h}^{m}=\pi_{h}\left(\operatorname{sgn}_{\varepsilon}\left(\tilde{\phi}_{h}^{n+1}\right) \frac{\mathbf{g}_{h}^{m}}{\left|\mathbf{g}_{h}^{m}\right|}\right)
$$

Finally, the discrete version of the redistance algorithm writes also explicitly:

$$
\psi_{h}^{m+1}= \begin{cases}\psi_{h}^{m} & \text { when }\left|\tilde{\phi}_{h}^{n}\right|<\varepsilon \\ \pi_{h}\left\{\psi_{h}^{m} \circ X_{\mathbf{v}_{\varepsilon}}^{m}+\Delta \tau \operatorname{sgn}_{\varepsilon}\left(\tilde{\phi}_{h}^{n}\right)\left(1-\left|\nabla \psi_{h}^{m}\right|\right)\right\} & \text { otherwise }\end{cases}
$$

\subsubsection{Improvement of the area and perimeter conservations}

The overall coupling method is described in Algorithm 1.

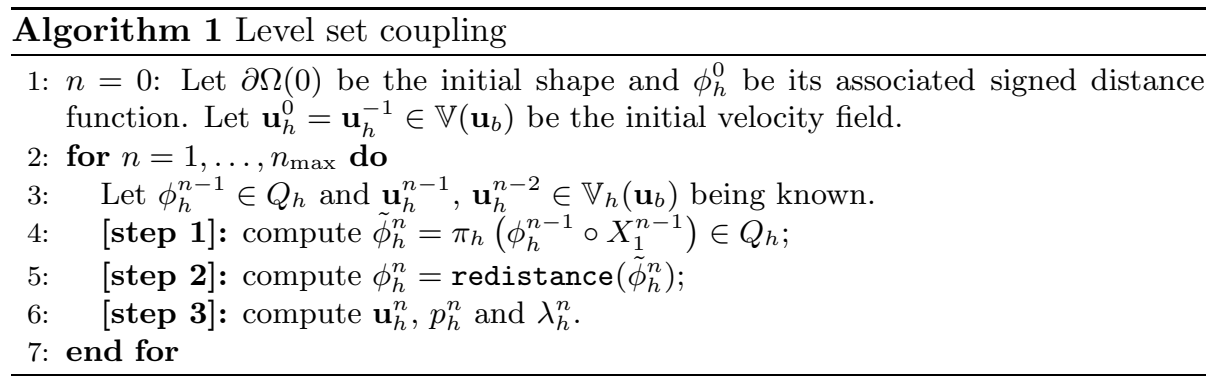

In this section we present a numerical simulation to illustrate the features of the numerical method. We choose $R e=10^{-3}, C a=10^{3}, \alpha=1 / 10, \tau=0.81$ and a viscosity ratio $\beta=50$. Fig. 2 plots the evolution of the relative error in vesicle area and perimeter. Observe that, after few iterations, the error becomes higher than $10 \%$ of the reference vesicle area and perimeter: this error completely changes the vesicle shape, that evolves to a circular one. The algorithm must be modified in order to improve the area and perimeter conservation. The 


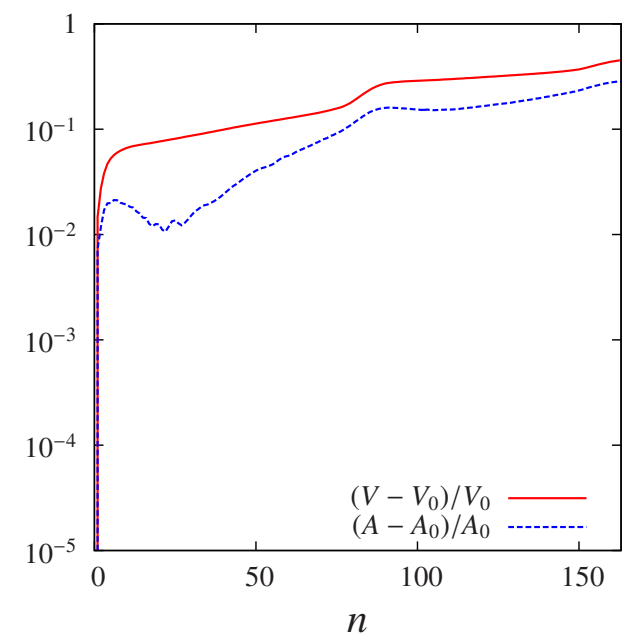

Figure 2: Relative errors for vesicle area and perimeter, without improving conservation. Computations for $h=5.3 \times 10^{-2}, \varepsilon=2.5 h, \Delta t=3 \times 10^{-2}$ and $\tau=0.81$.

problem of advection (4a) is substituted by the equivalent system:

$$
\begin{aligned}
& \left.\frac{\partial \phi}{\partial t}+\left[\mathbf{u}+\left(p_{*}+\lambda_{*} f\right) \mathbf{n}\right] \cdot \nabla \phi \quad=0 \quad \text { a.e }(t, x) \in\right] 0,+\infty[\times \Lambda, \\
& \left.\frac{\mathrm{d}}{\mathrm{d} t} \int_{\Lambda}(1-\mathscr{H}(\phi)) \mathrm{d} x \quad=0 \quad \forall t \in\right] 0,+\infty[, \\
& \left.\frac{\mathrm{d}}{\mathrm{d} t} \int_{\partial \Omega} \mathrm{d} s=0 \quad \forall t \in\right] 0,+\infty[,
\end{aligned}
$$

where $p_{*}$ and $\lambda_{*}$ are two global Lagrange multipliers associated to two additional constraints for area and perimeter preservation. This system leads, after time discretization, to a modified and more robust scheme, with a modified advection field $\mathbf{u}_{*}=\mathbf{u}+\left(p_{*}+\lambda_{*} f\right) \mathbf{n}$. The variation of area $V(t)$ at time $t^{n}$ writes:

$$
\frac{\mathrm{d} V}{\mathrm{~d} t}\left(t^{n}\right)=\left[\frac{\mathrm{d}}{\mathrm{d} t} \int_{\Lambda}(1-\mathscr{H}(\phi)) \mathrm{d} x\right]_{t=t^{n}}=\frac{V^{n}-V^{n-1}}{\Delta t}+\mathcal{O}(\Delta t),
$$

where $V^{n-1}=\int_{\Omega^{n-1}} \mathrm{~d} x$ is known and we want to impose that $V^{n}=V_{0}$ the initial area, in order to avoid the previous area error accumulation. Conversely, the variation of the perimeter $A(t)$ at time $t^{n}$ expresses:

$$
\frac{\mathrm{d} A}{\mathrm{~d} t}\left(t^{n}\right)=\left[\frac{\mathrm{d}}{\mathrm{d} t} \int_{\partial \Omega} \mathrm{d} s\right]_{t=t^{n}}=\frac{A^{n}-A^{n-1}}{\Delta t}+\mathcal{O}(\Delta t)
$$

where $A^{n-1}=\int_{\partial \Omega^{n-1}} \mathrm{~d} s$ is known and we want to impose that $A^{n}=A_{0}$ the initial perimeter. Combining (21a) and (21b), we obtain:

$$
\frac{\mathrm{d}}{\mathrm{d} t} \int_{\Lambda}(1-\mathscr{H}(\phi)) \mathrm{d} x=-\int_{\Lambda} \frac{\partial \phi}{\partial t} \delta(\phi) \mathrm{d} x=-\int_{\partial \Omega} \frac{1}{|\nabla \phi|} \frac{\partial \phi}{\partial t} \mathrm{~d} s=\int_{\partial \Omega} \frac{1}{|\nabla \phi|} \mathbf{u}_{*} \cdot \nabla \phi \mathrm{d} s .
$$


Recall that, for any function $\varphi$ and vector field $\mathbf{v}$, the Reynolds formula on a surface $\partial \Omega$ writes:

$$
\frac{\mathrm{d}}{\mathrm{d} t} \int_{\partial \Omega} f \mathrm{~d} s=\int_{\partial \Omega} \frac{\mathrm{d} f}{\mathrm{~d} t}+\nabla \cdot(f \mathbf{u})-f(\nabla \mathbf{u} \cdot \mathbf{n}) \cdot \mathbf{n} \mathrm{d} s .
$$

With $\varphi=1$ and $\mathbf{v}=\mathbf{u}_{*}$, and using the Green formula (10), we get successively:

$$
\frac{\mathrm{d}}{\mathrm{d} t} \int_{\partial \Omega} \mathrm{d} s=\int_{\partial \Omega} \operatorname{div}_{s} \mathbf{u}_{*} \mathrm{~d} s=\int_{\partial \Omega} H \mathbf{u}_{*} \cdot \mathbf{n} \mathrm{d} s .
$$

At time $t^{n}$, replacing $\mathbf{u}_{*}^{n}$ by $\mathbf{u}^{n}+\left(p_{*}^{n}+\lambda_{*}^{n} f\right) \mathbf{n}^{n}$ in (22)-(23) and using (24)-(26), we obtain the following linear system with two unknowns $\left(p_{*}^{n}, \lambda_{*}^{n}\right) \in \mathbb{R}^{2}$ :

$$
\begin{aligned}
p_{*}^{n} \int_{\partial \Omega} \mathrm{d} s+\lambda_{*}^{n} \int_{\partial \Omega} f \mathrm{~d} s & =\frac{V_{0}-\int_{\Omega^{n}} \mathrm{~d} x}{\Delta t}-\int_{\partial \Omega} \mathbf{u} \cdot \mathbf{n} \mathrm{d} s, \\
p_{*}^{n} \int_{\partial \Omega} H d s+\lambda_{*}^{n} \int_{\partial \Omega} H f \mathrm{~d} s & =\frac{A_{0}-\int_{\partial \Omega^{n}} \mathrm{~d} s}{\Delta t}-\int_{\partial \Omega} H \mathbf{u} \cdot \mathbf{n} \mathrm{d} s .
\end{aligned}
$$

Choosing $f$ a non-constant function ensure that this system is well-posed. In our simulations, we use $f\left(x_{1}, x_{2}\right)=2 x_{1}^{2}+x_{2}^{2}$. The influence of this function and the evolution of Lagrange multipliers will be discussed in section 4.1.

\subsubsection{Improvement by mesh adaptation}

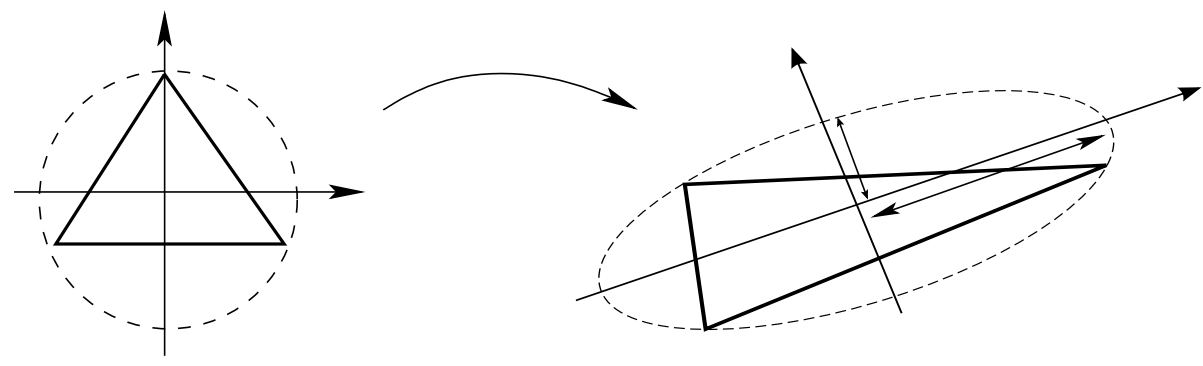

Figure 3: Transformation from the reference element $\hat{K}$ to any triangle $K$.

A way to adapt the mesh to the computation of a governing field is to equidistribute its interpolation error, i.e. to make it constant over all triangles and in the directions of maximal and minimal stretching and to adjust the maximal and minimal directions of stretching to others of maximal and minimal error. Our approach bases on the bidirectional anisotropic mesh generator bamg developed by F. Hecht [24] (see also [26, 27, 51]), together with the choice of a particular metric, specific to our time-dependent level set problem.

For any triangle $K$ of the mesh $\mathcal{T}_{h}$ at time $t$, let $T_{K}$ be the affine transformation which maps the reference triangle $\hat{K}$ into $K$ (see Fig. 3):

$$
\begin{aligned}
T_{K}: \hat{K} & \longrightarrow K \\
\hat{x} & \longmapsto \mathbf{x}=T_{K}(\hat{\mathbf{x}})=M_{K} \hat{\mathbf{x}}+\mathbf{t}_{K} .
\end{aligned}
$$


where $M_{K}$ is the Jacobian of $T_{K}$. Notice that $M_{K}$ is unsymmetric and invertible, otherwise $K$ would be flat. Thus, $M_{K}$ admits a singular value decomposition (for SVD, see [23, p. 69]): $M_{K}=R_{K}^{T} \Lambda_{K} P_{K}$, where $R_{K}$ and $P_{K}$ are orthogonal and where $\Lambda_{K}$ is diagonal with positive entries. The choice of the reference triangle $\hat{K}$ is not unique. It is common practice to choose as $\hat{K}$ the right triangle $\left\{\left(x_{1}, x_{2}\right), x_{1}>0, x_{2}>O, x_{1}+x_{2}<1\right\}$. For mesh generation and adaption purposes, an equilateral triangle, inscribed in the unit circle, is often preferred [20]. Since $\hat{x}=M_{K}^{-1}\left(x-t_{K}\right)$, the unit circle equation $\hat{x}^{T} \hat{x}=1$ becomes:

$$
1=\left(x-t_{K}\right)^{T} M_{K}^{-T} M_{K}^{-1}\left(x-t_{K}\right)=\left(x-t_{K}\right)^{T} R_{K}^{T} \Lambda_{K}^{-2} R_{K}\left(x-t_{K}\right)
$$

This is the equation of an ellipse containing $K$ (see Fig. 3 ).

Following [24], our choice of the metric is based on the Hessian tensor of a specific governing field $\chi$, for which we aim at decreasing the interpolation error. The interpolation error in the direction $\mathbf{v} \in \mathbb{R}^{2}$ is given by:

$$
e_{K, \mathbf{v}}=h_{K, \mathbf{v}}^{2}\left\|\frac{\partial^{2} \chi}{\partial \mathbf{v}^{2}}\right\| \text { on } K
$$

where $h_{K, \mathbf{v}}$ denotes the length of $K$ in the direction $\mathbf{v}$ and $\frac{\partial^{2} \chi}{\partial \mathbf{v}^{2}}=\mathbf{v}^{T} \nabla \nabla \chi \mathbf{v}$, and $\nabla \nabla \chi$ is the Hessian matrix of $\chi$.
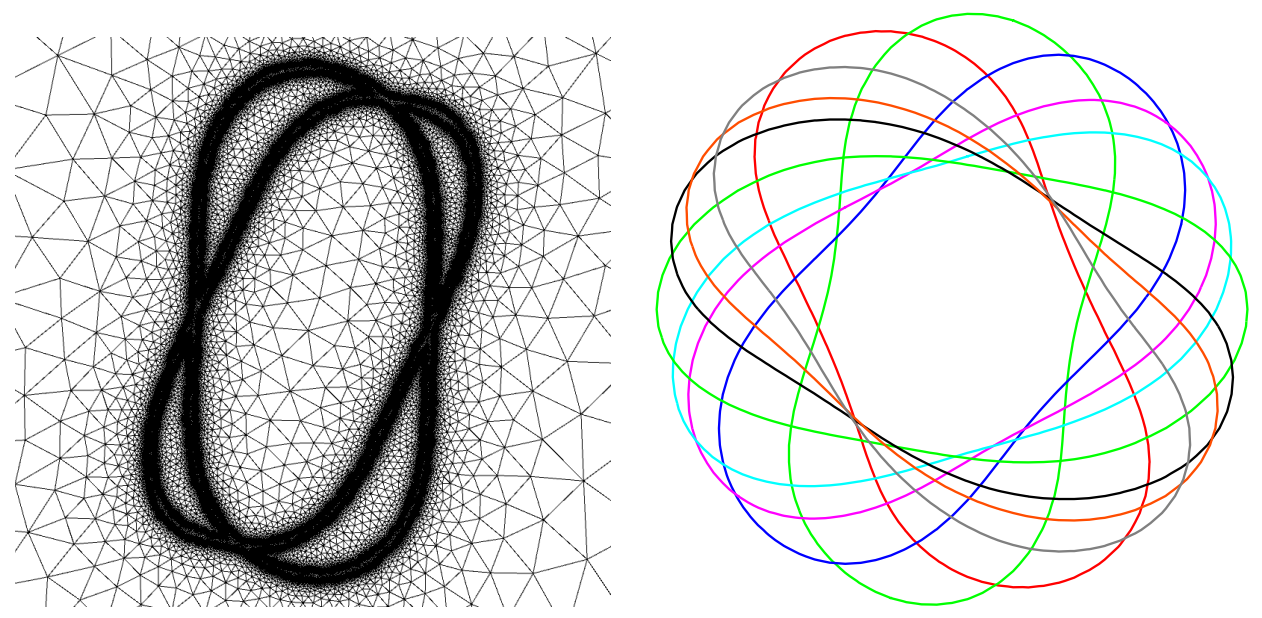

Figure 4: (left) Zoom on the adapted mesh ; (right) Vesicle tumbling under a linear shear flow for $R e=10^{-3}, C a=10^{4}, \alpha=1 / 4, \beta=20$ and $\gamma=0.89$. The shapes are shown for $t=k T_{p} / 14, k \in\{1,2,3,4,5,8,11,12,13\}$, where $T_{p}=10.3$ is the tumbling period.

By adjusting the directional sizes $h_{K, \mathbf{v}}$ of $K$ for each eigenvector of the Hessian matrix and each element $K$, the local directional interpolation errors can be equidistributed on the whole domain. An adaptation loop is required in order to guarantee the convergence of both the approximation of $\chi$ and its corresponding mesh. In order to adapt the mesh to the vesicle boundary $\partial \Omega^{n}$ at each time 
step $t_{n}$, the governing field $\chi=\delta_{\varepsilon}\left(\phi^{n}\right)+\delta_{\varepsilon}\left(\phi^{n-1}\right)$ has been chosen for the adaptation loop. For a uniform mesh, the regularization parameter used for the computation of integrals over $\partial \Omega$ is chosen as proportional to the element size: $\varepsilon=2 h$. This choice is extended to a non-uniform mesh with a non-constant $\varepsilon(x), x \in \Lambda$, that is proportional to an average value of the local mesh size: $\varepsilon(x)=2 \sqrt{2} \operatorname{meas}(K)^{\frac{1}{2}}$, for all $x \in K$. Fig. 4.a shows a zoom on the adapted mesh at the end of the adaptation loop, where both the contours of $\partial \Omega^{n-1}$ and $\partial \Omega^{n}$ are captured. Fig. 4.b represents the vesicle boundary evolution, as computed by the present auto-adaptive procedure.

\subsection{Implicit time splitting algorithm}

In order to deal efficiently with the highly nonlinear term expressing the Helfrich force, a fixed point algorithm variant is introduced between the two main steps of the previous algorithm:

(i) Firstly, the nonlinear dynamics is solved for fixed level set function and membrane geometry description;

(ii) Secondly, the advection and redistance subproblem are solved for a fixed fluid velocity.

This fixed point iteration is repeated until the relative error is less than a given tolerance $\epsilon_{\mathrm{fp}}$, choosen here as $10^{-6}$ for the practical computations. This strategy has the advantage to split the problem into a succession of two simpler, linear and more standard subproblems, while maintaining a robust implicit algorithm. The overall implicit method is detailed in Algorithm 2. 


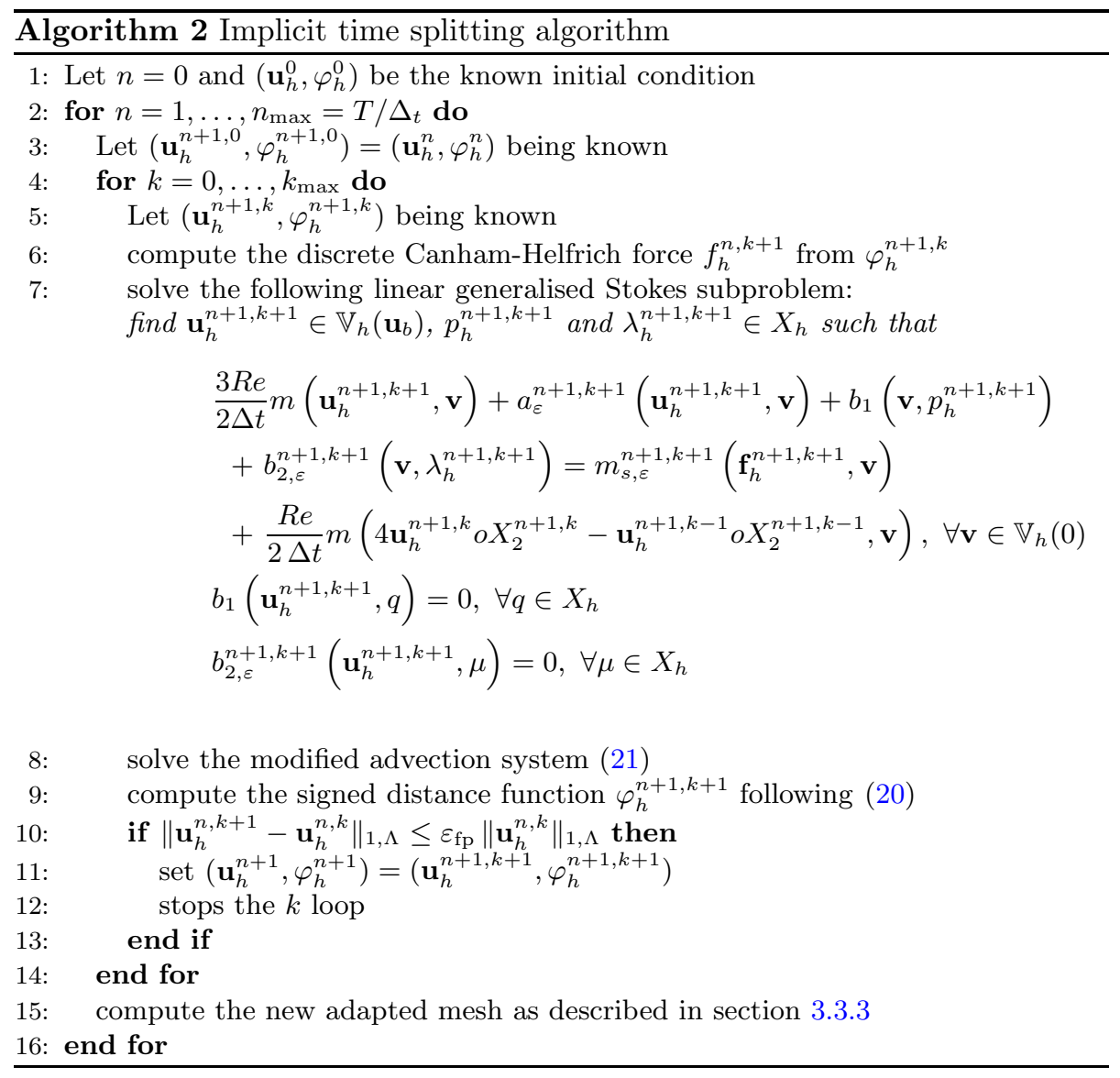

\section{Numerical results}

In this section, we provide several numerical tests carried out with the finite element level set approach previously described. The algorithms employed in the simulations have been implemented using a free software, the finite element library Rheolef [51].

Biophysical applications, mainly the prediction of vesicles behavior in small blood vessels, is the scoop of this paper. Simulations show, in accord with literature, that two flow regimes exist: a steady-state tank-treading regime where the vesicle assumes a steady-state shape and its inclination angle remains constant with time, while the fluid membrane treads as a tank and the internal fluid follows this rotation. The second regime is a periodic tumbling one, where the vesicle shape rotates. The transition between the two regimes for a vesicle of fixed reduced area $\gamma$ happens at a critical viscosity ratio between the inside and outside fluid, beyond which the vesicle tumbles. In order to validate the proposed method, we compared our results with available numerical data, i.e. 
for small Reynolds number. We have determined the transition line separating the two regimes. These results are also founded to be in good agreement with the phase field method presented in [5] (see also Fig. 2 in [38] for a comparison). Note that both the tank treading and tumbling motions are observed experimentally (see e.g. Kantsler and al. [30]).

In order to make a validation of our computations, we follow the experiments of Vitkova and al. [54] with vesicles under shear flow. This is a typical situation in microfluidic devices and the viscous forces are dominant over the inertial ones: the flow is almost laminar, and no turbulence can be observed, at least in the absence of vesicle. The considered physiological parameters are

$$
\begin{aligned}
& R_{0} \approx 5 \times 10^{-5} \mathrm{~m}, \quad \rho \approx 10^{3} \quad \mathrm{~kg} / \mathrm{m}^{3} \text {, } \\
& L \approx 10^{-3} \mathrm{~m}, \quad \eta_{0} \in\left[5 \times 10^{-4}, 0.2\right] \mathrm{kg} . \mathrm{s}^{-1} \cdot \mathrm{m}^{-1} \text {, } \\
& k \approx 10^{-19} \mathrm{~J} \text {. }
\end{aligned}
$$

The shear velocity at the wall $V$ is between $1.2 \times 10^{-2}$ and $0.12 \mathrm{~m} / \mathrm{s}$. Following the experimental values, we consider in a first step these dimensionless parameter ranges: $R e \in\left[1.5 \times 10^{-9}, 1.5 \times 10^{-4}\right]$ and $C a \in\left[3 \times 10^{3}, 3 \times 10^{6}\right]$. Besisdes, vesicles are deflated and correspond to $\gamma \in[0.92,0.99]$. However, Vitkova and al. [54] use confined vesicles in a pipe with a length equal to $1 \mathrm{~mm}$. This leads to a confinement ratio equal to $1 / 20$. The influence of this parameter is studied in the following and depends on considered meshes. Lastly, the viscosity ratio $\beta$ change around a critical value associated to a stability transition, and it is taken usually smaller than $20 \mathrm{~kg} \cdot \mathrm{s}^{-1} \cdot \mathrm{m}^{-1}$.
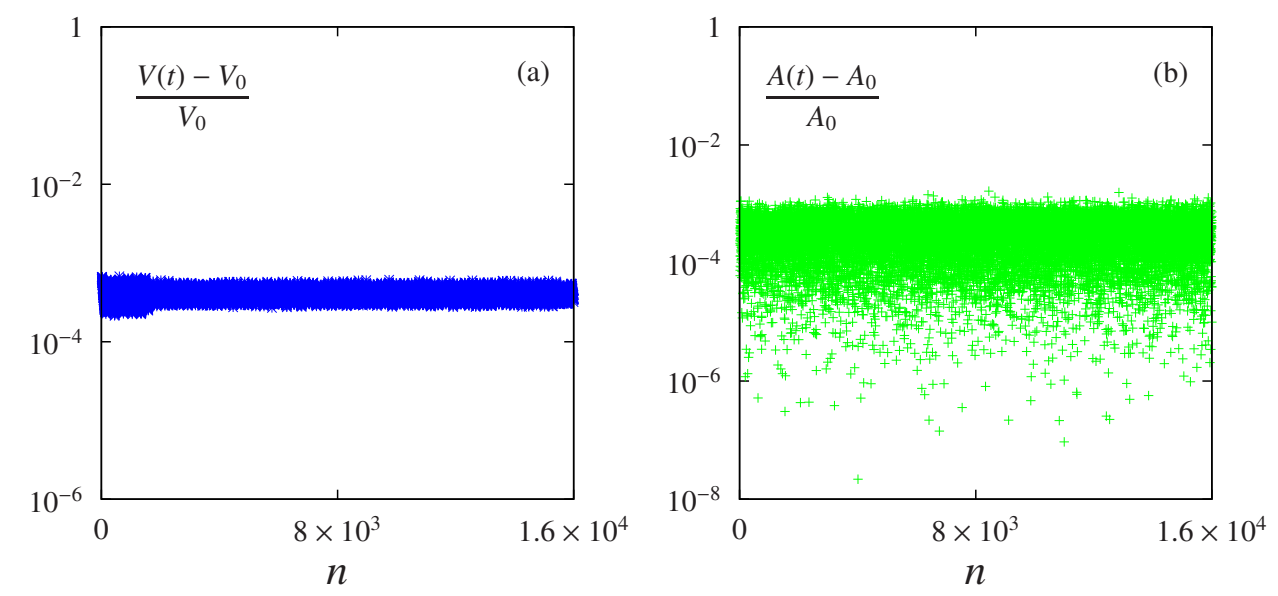

Figure 5: Relative errors in area and perimeter, after conservation improvement: (a) the vesicle area error; (b) the vesicle perimeter error. 


\subsection{Vesicles in the tumbling mode}

In this section, we restrict ourselves to small reynolds numbers. , and we consider the following parameters: $R e=10^{-4}, C a=10^{3}, \gamma=0.89$ and $\Delta t=2.5 \times$ $10^{-3}$. Besides, actual computations use confinements in the range $[0.2,0.5]$ when using regular meshes, and reach confinements up to $1 / 12$ in the case of adapted meshes. We notice that, in this section, we choose the viscosity ratio $\beta$ such that the vesicle follows a tumbling regime.

Mass preservation. - First, let us check the improvement of the area and perimeter conservation, as introduced in the procedure of the previous section. Computations are firstly performed with $\alpha=1 / 9$, and we plot in Fig. 5 the evolution of the relative errors $\left(V-V_{0}\right) / V_{0}$ and $\left(A-A_{0}\right) / A_{0}$. We observe that, over a

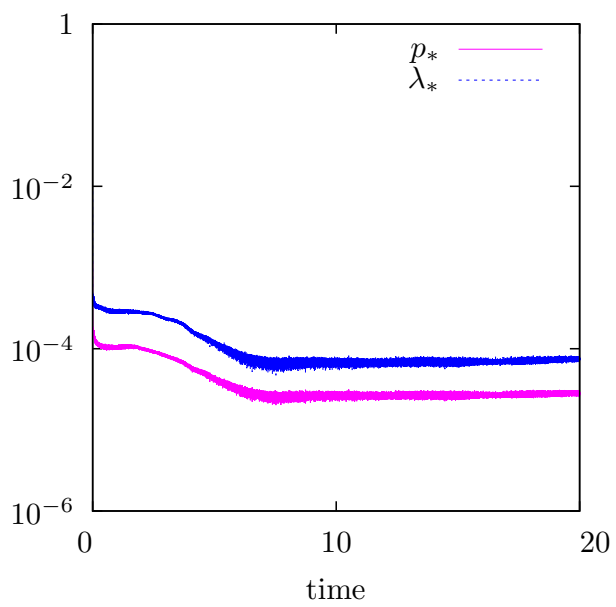

Figure 6: Evolution of the Lagrange multipliers for the tumbling of a vesicle with $\gamma=0.89$ in a flow given by $R e=10^{-4}$ and $C a=10^{3}$.

duration of 80 periods of tumbling, both the relative errors in area and perimeter remain bounded by $10^{-3}$. The improvement of the conservation, based on Lagrange multipliers, is clearly shown by a comparison with the previous computations in Fig. 2, where the errors diverge after two periods in the tumbling regime. Consequently, we emphasize that the Lagrange multipliers technique is strongly needed in order to deal with the mass lost that represents the major problem of Eulerian methods. Additionally, the influence of these parameters on the vesicle dynamics has to be investigated deeply. To explore the influence of $\lambda_{*}$ and $p_{*}$, we plot the evolution of these parameters in time. From Fig. 6, the two Lagrange multipliers appear to remain bounded and very small, and we can conclude that the influence they have on the dynamics can be neglected, especially when compared to a change in mass or area. This change will have significantly a stronger effect on the dynamics. 

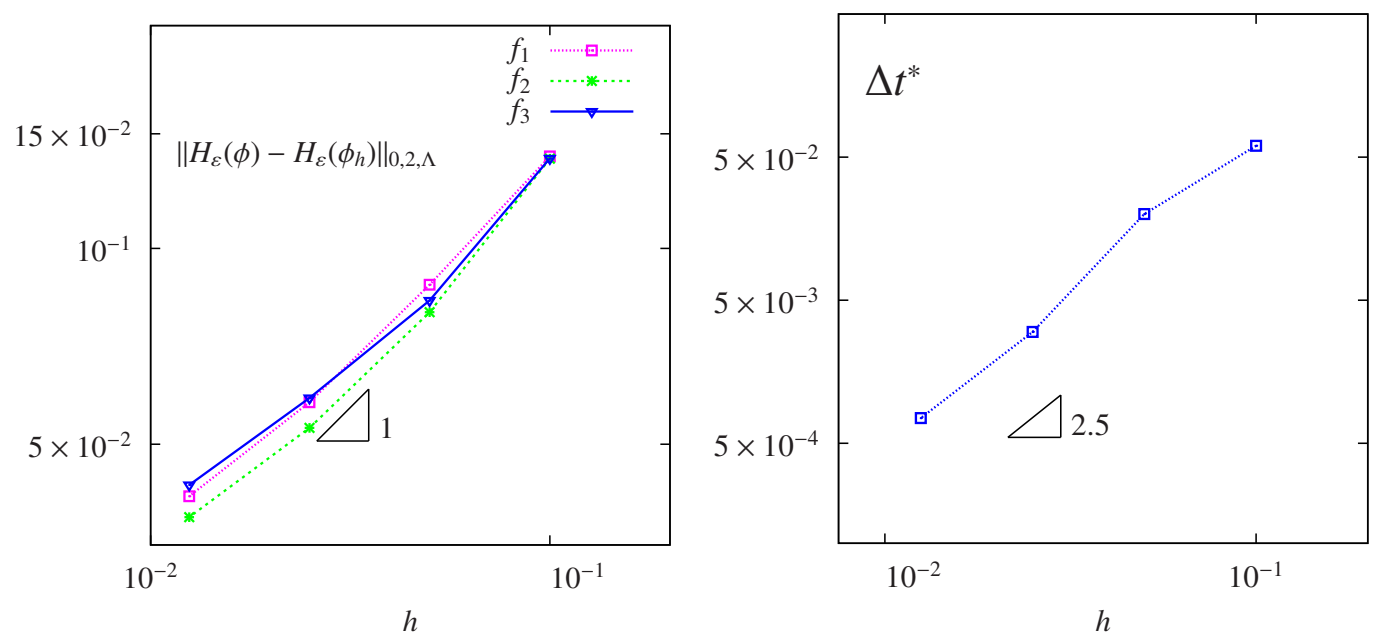

Figure 7: (a) Convergence properties for three different expressions of function $f$. (b) Critical value of the time step in log-log scale for different mesh steps.

Dependance on the choice of $f$. - We investigate the effect of the choice of the function $f\left(x_{1}, x_{2}\right)$ (see (21a)) on the computation of the solution. We use three expressions of $f: f_{1}\left(x_{1}, x_{2}\right)=2 x_{1}^{2}+x_{2}^{2}, f_{2}\left(x_{1}, x_{2}\right)=2 x_{1}^{2}+5 x_{2}$ and $f_{3}\left(x_{1}, x_{2}\right)=3 x_{1}$. The dimensionless parameters are $R e=0.1, C a=10^{4}$ and $\beta=75$ and we study the dynamics of vesicle with $\gamma=1$. Under simple shear flow; it keeps a circular shape and we compute the errors between the numerical and the exact solution for the time interval $] 0,2 s[$ in Fig. 7.a. The error upon the geometry is expressed by the $L^{2}$ norm of $\mathscr{H}_{\varepsilon}(\phi)-\mathscr{H}_{\varepsilon}\left(\phi_{h}\right)$ where $\phi$ and $\phi_{h}$ are respectively the exact and the computed level set functions, and $\mathscr{H}$ denotes the regularized Heaviside function (see section 3.2.3).

Results show that the error decreases versus the mesh size, and the choice of $f$ doesn't affect the convergence of the method. Moreover, the plot suggests a $\mathcal{O}(h)$ convergence of the predicted geometry versus the mesh size. after two tumbling periods.

Dependance upon the time step. - From the computational cost wiepoint, the choice of the time step is very important particularly because the method has to be used in three-dimensional case in forthcoming works. Therefore, we consider in this section only a regular mesh, and we investigate how does the chosen time step compares to the space discretization.

Firstly, we consider the case when the algorithm is semi-implicit; this corresponds to a fixed point loop in Algorithm 2 that is stopped after the first computation, i.e. $k_{\max }=1$. Due to the moving interface and the computation of the Helfrich forces, there exists a critical value of the time step $\Delta t^{*}$ such that, for time steps beyond this threshold value $\Delta t>\Delta t^{*}$, the algorithm is not stable. We do computations using the following parameters: $R e=1, C a=10^{4}$, 
$\beta=100$ and $\gamma=0.95$. Then, we plot in Fig. 7.b the critical value $\Delta t^{*}$ for different values of the mesh size $h$ and we observe that the slope of the regression line is about 2.5: this suggests that $\Delta t^{*}$ behaves like $h^{2.5}$. In fact, this time step limitation is related to the case of an explicit scheme, and the use of the implicit algorithm appears therefore to be of major importance.

Secondly, we focus on the fix point algorithm 2. Computations show that there is no more limitation on the choice of the time step but in practice, we consider usually $\Delta t \in[0.01,0.1]$. However, the linear system has to be solved inside each fix point loop, and, for a time step $\Delta t=0.01$, we need usually between five and seven sub-iterations until we reach the tolerance $\epsilon_{f p}$, which garantees stability for the coupling algorithm.

Dependance on the confinement. - In this subsection, we investigate the ef-
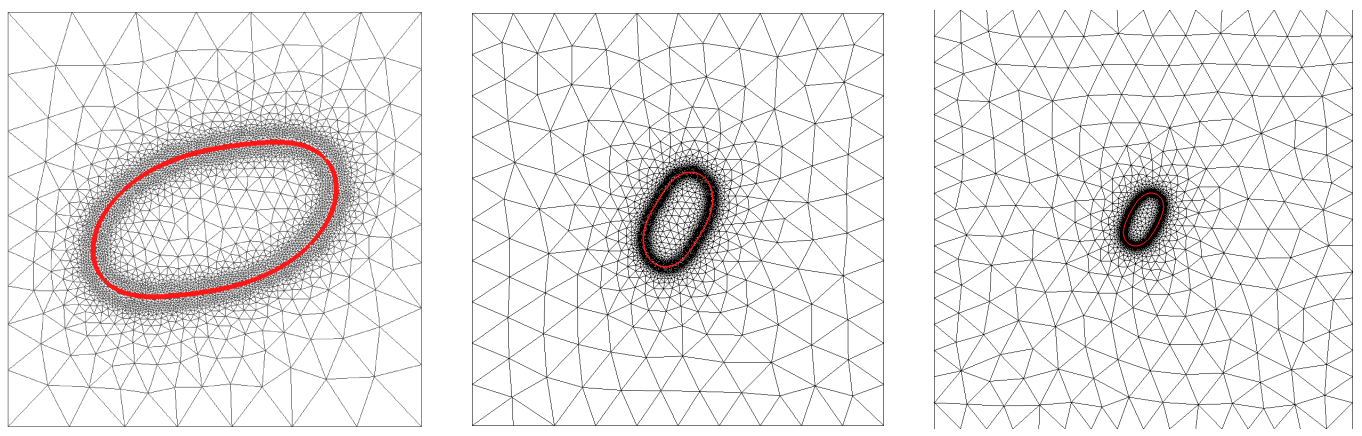

Figure 8: Adapted meshes used for the study of the effect of the confinement $\alpha$ : from left to right: $\alpha=1 / 2,1 / 5$ and $1 / 9$.

fect of the confinement $\alpha$ on the vesicle tumbling regime: a recirculation flow around the vesicle membrane can change deeply the dynamics behaviour of the membrane. Adapted meshes, that capture the vesicle boundary are shown in Fig. 8 for different confinements. Fig. 9 plots the evolution of the tumbling dimensionless period, denoted by $T_{p}$, versus $1 / \alpha$. As expected, these results show that results depend strongly on the confinement $\alpha$ when $\alpha$ is close to one, i.e. when the vesicle is confined, while the tumbling period becomes independent for small $\alpha$ values. In the simulations presented in the rest of the paper, we choose $\alpha=1 / 4$ : this choice guaranties that the dynamics depends weekly upon the confinement.

Let us denote by $\theta(t)$ the inclination angle measured counterclockwise from the positive $x_{1}$ semi-axis. The numerical computation of the inclination angle $\theta(t)$ for an arbitrary shape $\Omega(t)$ is reported in appendix AppendixD. The vesicle reaches a periodic regime after about ten periods of tumbling, and the inclination angle $\theta(t)$ follows a periodic regime. We set a simulation using the following dimensionless parameters: $R e=10^{-4}, C a=10^{3}, \alpha=1 / 4, \beta=20$ and $\gamma=0.82$, and we observe some Lissajous representations that are suitable for periodic 


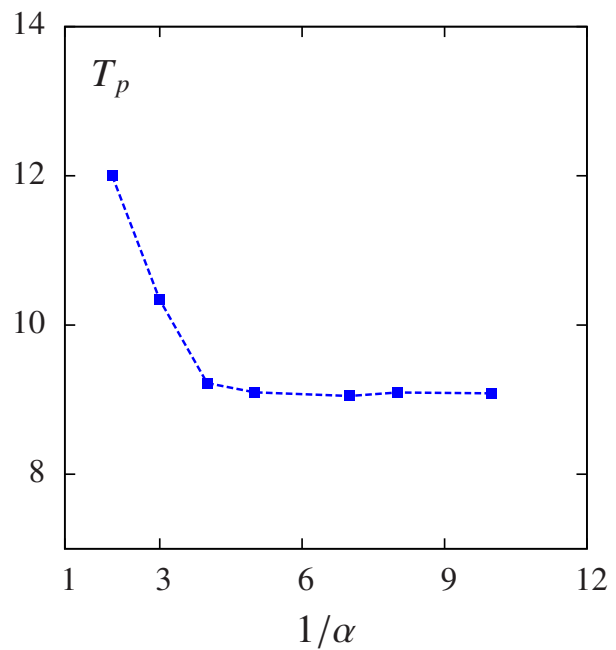

Figure 9: Period $T_{p}$ in the tumbling regime with respect to $1 / \alpha$. The simulation corresponds to: $R e=10^{-4}, C a=10^{3}, \beta=20$ and $\gamma=0.82$.
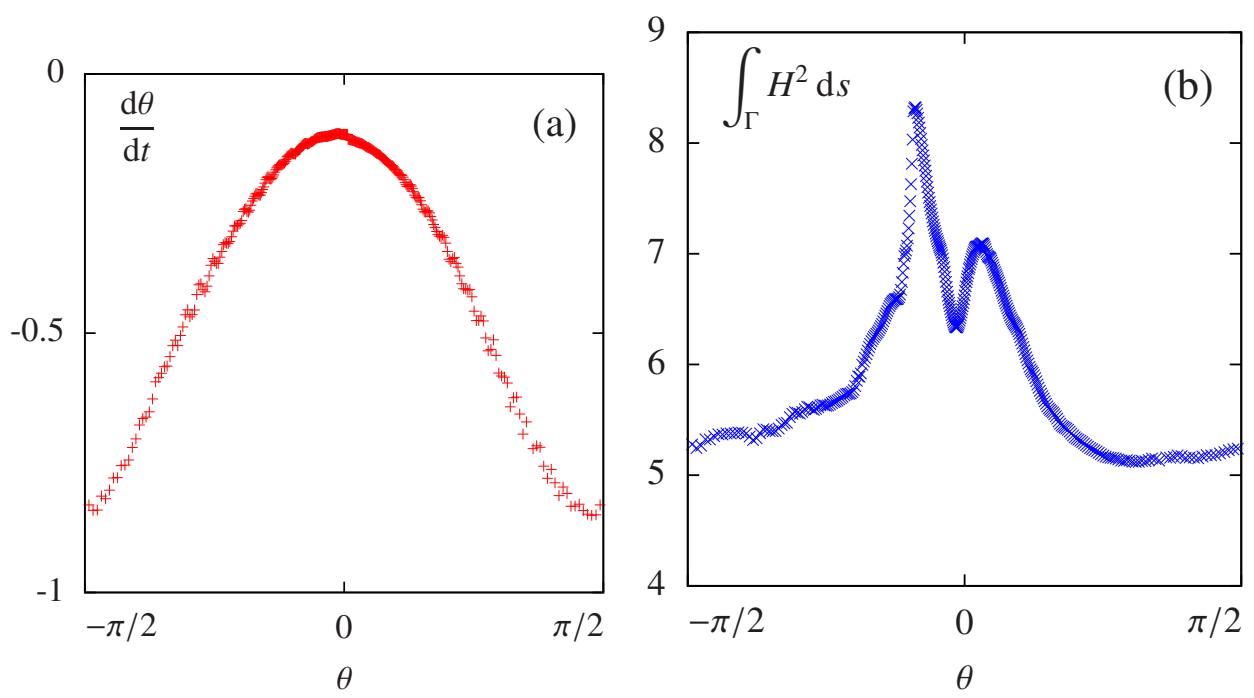

Figure 10: Lissajous curves in the tumbling regime: (a) the angular velocity $\frac{d \theta}{d t} v s \quad \theta$ and (b) the Canham-Helfrich energy vs $\theta$.

phenomenas. Once the periodic regime is well established, we plot in Fig. 10.a the angular velocity $\frac{d \theta}{d t}$ with respect to $\theta$ : we observe that the angular velocity is minimal when $\theta=0$, i.e. when the vesicle is aligned with the horizontal axis, 
while its maximal when the vesicle is aligned vertically $(\theta= \pm \pi / 2)$. Fig. 10.b plots the evolution of the Canham-Helfrich energy with respect to $\theta$ : this energy reaches a global maximum when the vesicle is roughly aligned horizontally and, conversely, reaches a minimum when its roughly aligned vertically. Here, there is a small phase shift: the extrema of the energy are slightly in advance with the corresponding extrema of the angular velocity.

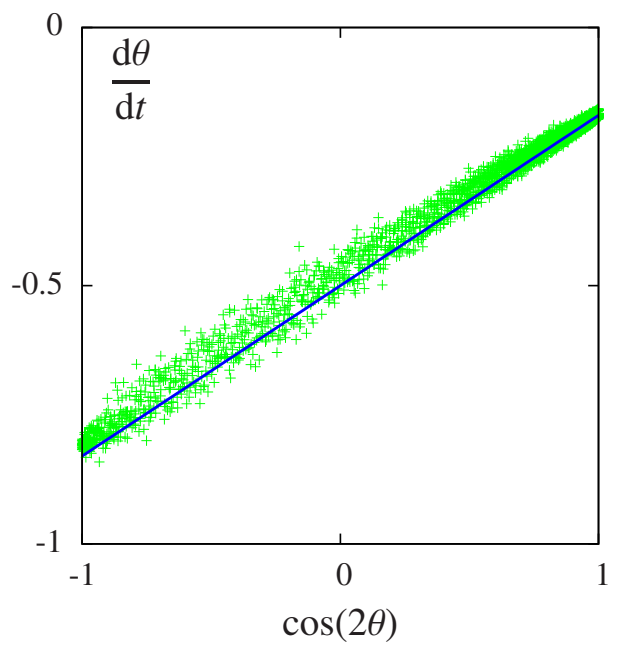

Figure 11: Evolution of the angular velocity $\frac{d \theta}{d t}$ in the tumbling regime with respect to $\cos (2 \theta)$. The dimensionless parameters of the computation are $R e=10^{-4}, C a=10^{4}, \alpha=1 / 9, \beta=50$ and $\gamma=0.84$. The linear regression suggests that $\frac{d \theta}{d t}=0.33 \cos (2 \theta)-0.5$, as indicated by the continuous line.

In order to study analytically the dynamics of vesicles, a rough analytical model was proposed in 1982 by Keller and Skalak [31]. This model incorporates a quasi-inextensible membrane, but vesicles were treated as undeformable liquid ellipsoids. Nevertheless, this model was able to reproduce the tumbling regime for reduced areas $\gamma$ near 1 (i.e. quasi-spherical shapes), for which the distance to inextensibility is weak. Keller and Skalak [31] showed that the ellipsoid motion is described by:

$$
\frac{\mathrm{d} \theta}{\mathrm{d} t}=-\frac{1}{2}+c(\gamma, \beta) \cos (2 \theta)
$$

where $c(\gamma, \beta)$ is a coefficient depending on the aspect ratio $\gamma$ and the viscosity ratio $\beta$. Fig. 11 plots $\frac{\mathrm{d} \theta}{\mathrm{d} t}$ versus $\cos (2 \theta)$. Observe the good correspondence with the affine behavior, as predicted by the Keller and Skalak theory. A linear regression on the numerical simulation data leads to the slope coefficient $c=$ 0.33 .

Dependance on the reduced area. - Let us turn to the effect of the reduced area 


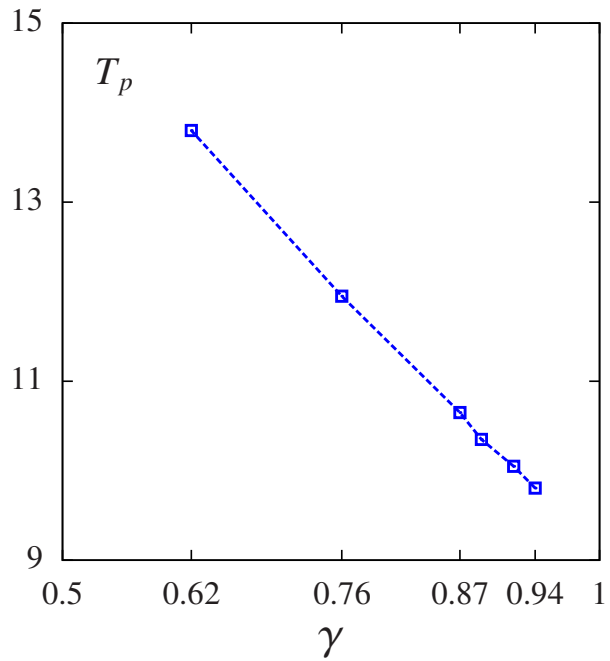

Figure 12: Tumbling regime: period $T_{p}$ vs the reduced area $\gamma$, for $R e=10^{-4}, C a=10^{3}$, $\alpha=1 / 4$ and $\beta=50$.

$\gamma$ on the period of tumbling $T_{p}$. We consider a vesicle with a viscosity ratio $\beta=50$ in a shear flow with a Reynolds number $R e=10^{-4}$ and a Capillarity number $C a=10^{3}$. Observe on Fig. 12 the quasi-linear dependence of $T_{p}$ upon $\gamma$. This behaviour can be interpreted as follow: when the vesicle is more circular (e.g. for high reduced area), it is less deformed during the tumbling dynamics and its rotational moement is easier. As a consequence, the tumbling period becomes smaller.

\subsection{The tank-treading regime}

When the viscosity ratio $\beta$ becomes smaller than a critical value, a transition to the tank-treading regime occurs. The fluid inside the vesicle is highly deformed and rotated, and the vesicle adopts a stationary boundary $\partial \Omega$. Fig. 13 plots the streamlines and the velocity fields on the vesicle membrane. Remark that, when the stationary regime is reached, the velocity field become tangential to the membrane, and then we observe that the tank treading movement becomes more appropriate to preserve the minimal energy state. Fig. 14 represents the vesicle orientation $\theta(t)$ : observe that it reaches rapidly a stationary value, denoted by $\theta^{*}$. Notice that the velocity is not vanishing along $\partial \Omega$ : the membrane continues to tread like a tank and the internal fluid follows this rotation.

Numerical validation. - In this paragraph, we study the convergence properties of the numerical solution versus both the mesh refinement $h$ and the confinement $\alpha$. For this purpose, we perform a shear flow simulation of vesicles with several reduced areas $\gamma$ in the tank-treading regime. The convergence properties are 

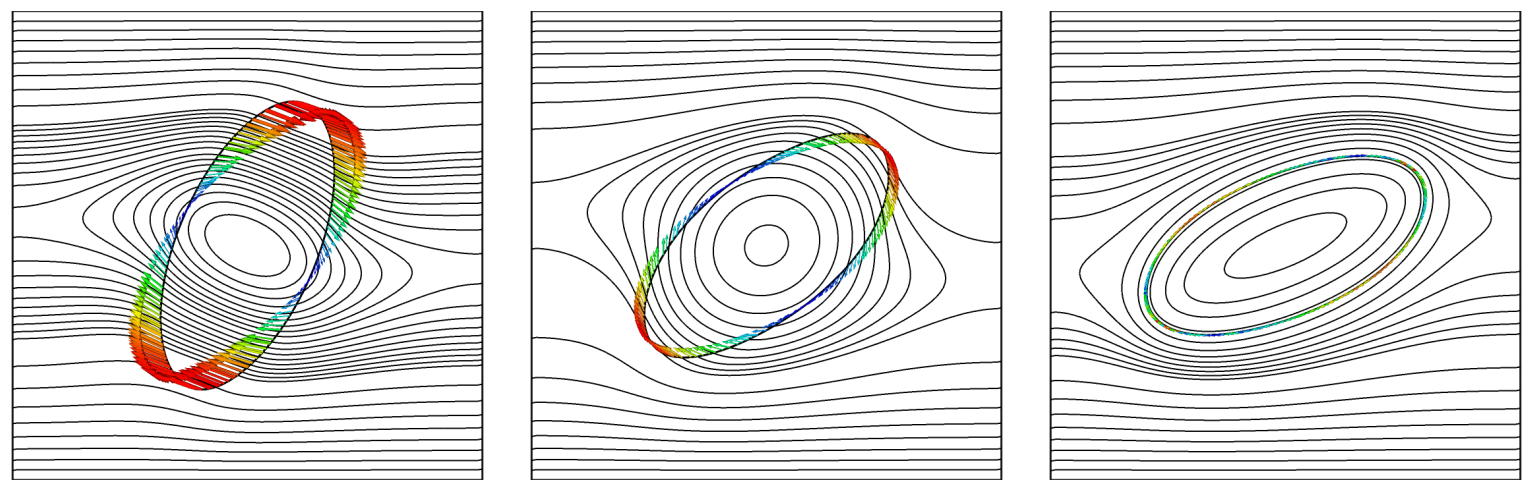

Figure 13: Tank-treading regime vs time for $R e=10^{-4}, C a=10^{4}, \alpha=1 / 2, \beta=1$ and $\gamma=0.84$ : streamlines lines and velocity field on the vesicle membrane $\partial \Omega$. Figures are, from left to right, at $t=k \Delta t, k \in\{60,120,1000\}$ and $\Delta t=2 \times 10^{-2}$.

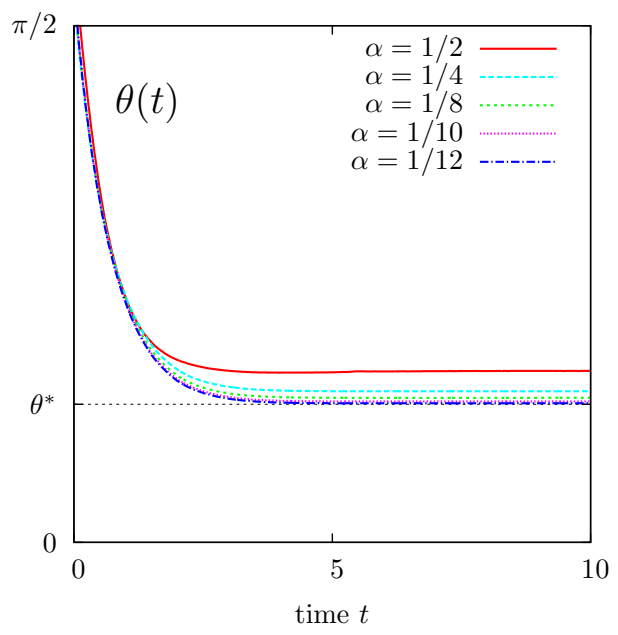

Figure 14: Tank-treading regime: time evolution of the tumbling inclination angle for various confinements $\alpha$ and $\gamma=0.89$.

evaluated by observing the dependence of the stready state inclination angle $\theta^{*}$ upon $h$ and $\alpha$.

Let us first consider the dependence upon mesh refinement, where a family of regular grids are considered. Fig 15 groups in a table the stationary angles $\theta_{h}^{*}$ obtained for decreasing values of $h$ and for various reduced areas $\gamma$. By extrapolation for $h=0$, we are able to compute an improved value of the stationary angle, denoted simply as $\theta^{*}$. Our extrapolation is based on a linear least square procedure, as implemented in [56]. Then, the error is estimated as $\theta_{h}^{*}-\theta^{*}$ and ploted on Fig. 15. Observe that the slope in logarithmic scale is of 


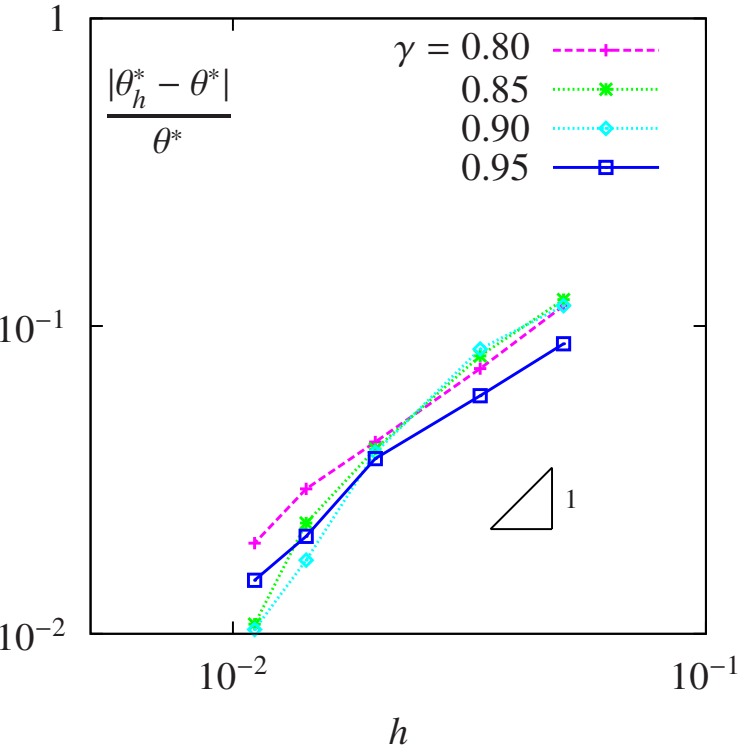

Figure 15: Tank-treading regime: convergence of the stationary angle $\theta^{*}$ versus the mesh refinement $h$

\begin{tabular}{|l||cccc|}
\hline $1 / \alpha$ & 0.8 & 0.85 & 0.9 & 0.95 \\
\hline \hline 2 & 0.4301 & 0.5472 & 0.6300 & 0.8212 \\
4 & 0.3951 & 0.4705 & 0.5546 & 0.7502 \\
8 & 0.3615 & 0.4409 & 0.5224 & 0.7115 \\
12 & 0.3518 & 0.4234 & 0.5183 & 0.7004 \\
extrapolation & 0.3412 & 0.4150 & 0.5114 & 0.6928 \\
\hline
\end{tabular}

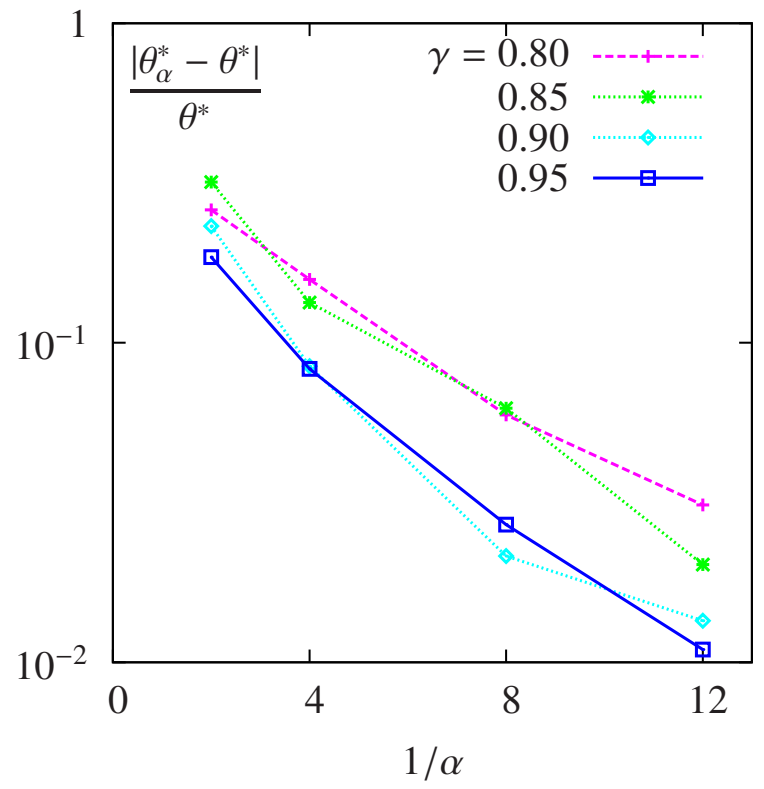

Figure 16: Tank-treading regime: convergence of the stationary angle $\theta^{*}$ versus the confinement $1 / \alpha$. 
about one, suggesting a $\mathcal{O}(h)$ convergence.

Figure 16 groups in a table the stationary angles $\theta_{\alpha}^{*}$ obtained for decreasing values of the confinement $\alpha$ and for various reduced areas $\gamma$. By extrapolating to $\alpha=0$, we compute an improved value of the stationary angle, denoted simply as $\theta^{*}$ and estimate the error $\theta_{\alpha}^{*}-\theta^{*}$. The plot on Fig. 16 suggests an exponential convergence of the stationary angle to a value associated to an unconfined vesicle.

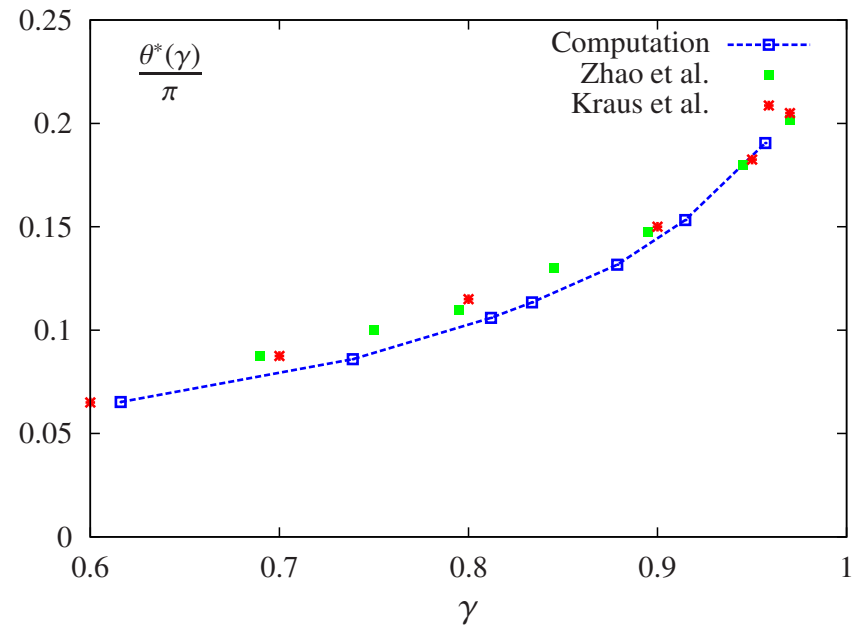

Figure 17: Inclination angle of the steady-state tank-treading regime with a viscosity ratio $\beta=1$. Comparative study for $C a=10$ with nemerical results of Zhao et al. (2009) [57] for $C a=9$ and Kraus et al. (1996) [33] for $C a=10$.

Experimental validation: comparaison with data in vitro and simulations. For accuracy raisons, we compare our results with those performed by Zhao et al. (2009) [57] and by Kraus et al. (1996) [33]. Then, in terms of physiological relevance, we compare with experiment measurments observed by Kantsler \& Steinberg (2005) [30]. Firstly, we study the dynamics of vesicles in the tank treading regime for vesicles with different reduced areas and we perform a quantitative comparaison with the results presented in [33] and [57]. Then, by modifying the viscosity ratio, we perform simulations for $\beta \in 1,2.7,5.4$ and we proceed to a comparative study in light of experimental observations in [30] and numerical results in [57]. In both simulations and data, we observe from Figure 17 and Figure 18, by plotting the dependence of $\theta^{*}$ upon $\gamma$, a close fit of the simulated vesicle motion with respect to the experiments and numerical data. Moreover, We notice that results for the inclination angle for very small values of $R e$ are consistent with the computational results of Salac and al. presented in Fig.17 in [49]. In summary, we obtain a satisfactory agreement between numerical results and measured data. 


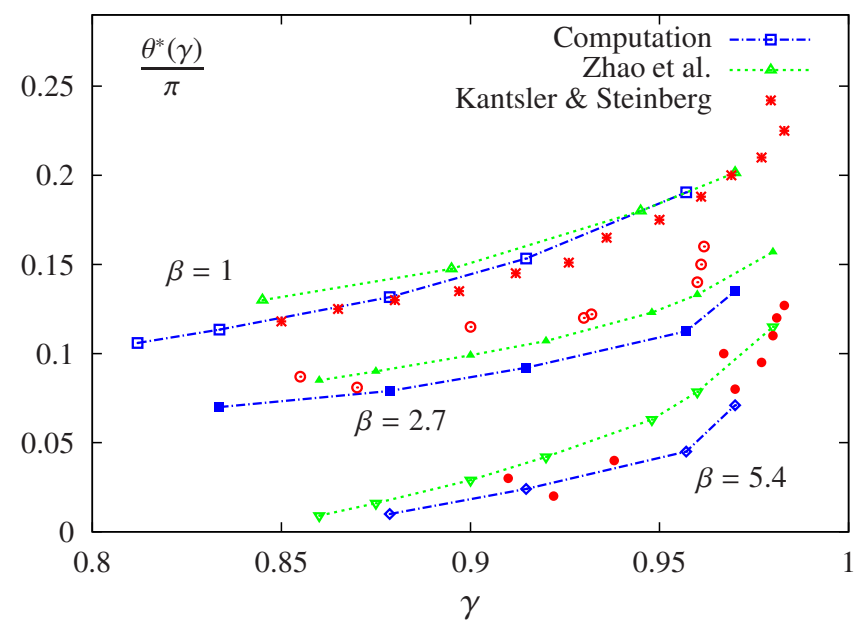

Figure 18: Inclination angle of the steady-state tank-treading regime with different viscosity ratio $\beta=1, \beta=2.7$ and $\beta=5.4$ : comparative study of our results (datched blue line with squares) with numerical results of Zhao et al. (2009) (dotted green line with triangular symbols), and experimental validation with the measurements of Kantsler \& Steinberg (2005) [30] (circular red point symbols).

\subsection{Effect of inertia}

An exhaustive study of the rheology of a vesicle in the presence of inertia has been carried out in this section. Although the basic behaviors had already been observed, the results shown in this part were nontrivial and not completely understood yet. In fact, we notice that the Reynolds number (at the scale of the $\mathrm{RBC}$ ) in the blood circulatory system is not always small enough for the Stokes limit to be valid. From a rough estimation in [38] it follows that the Reynolds number, particularly in the arterioles, evaluated at the scale of the RBC may be of order unity. By the same way, the radius of experimental vesicles used in laboratory experiments is of about $5 \times 10^{-5} \mathrm{~m}$ while it is possible to supervise vesicles with velocity of $0.1 \mathrm{~m} . \mathrm{s}^{-1}$ using rapid cameras; the estimation leads to $R e \approx 5$. Consequently, it follows from these estimations that the inertial effect is of the same order as the viscous one; it can no more be neglected and the prediction of vesicle behaviors should be studied for these magnitude of the Reynolds numbers. In the following, we find that a Reynolds number of order one can destroy completely the vesicle tumbling motion obtained in the Stokes regime. We plot in Fig. 20 the movement of the vesicle for a finite Reynolds value $R e=0.4$. At each time, we observe that the deformed shape of the vesicle differs a lot from the shape represented in Fig. 4.b and corresponding to small Reynolds numbers. We remark especially that the deformations are more important when the inclination angle is close to $\pi / 2$. Above a threshold value of the Reynolds number, computations reveal that the tumbling regime disappears in favor of tank treading regime, and the vesicle keeps then a constant inclination 
angle. We plot in Figs. 19.a and 19.b the angle $\theta(t)$ for $\gamma=0.82$, and we observe that the period $T_{p}$ increases with $R e$ until it reach a critical Reynolds number between 3.5 and 4 . For $R e>4$, the angle $\theta(t)$ becomes constant: the vesicle switch from a tumbling regime to a tank-treading one.
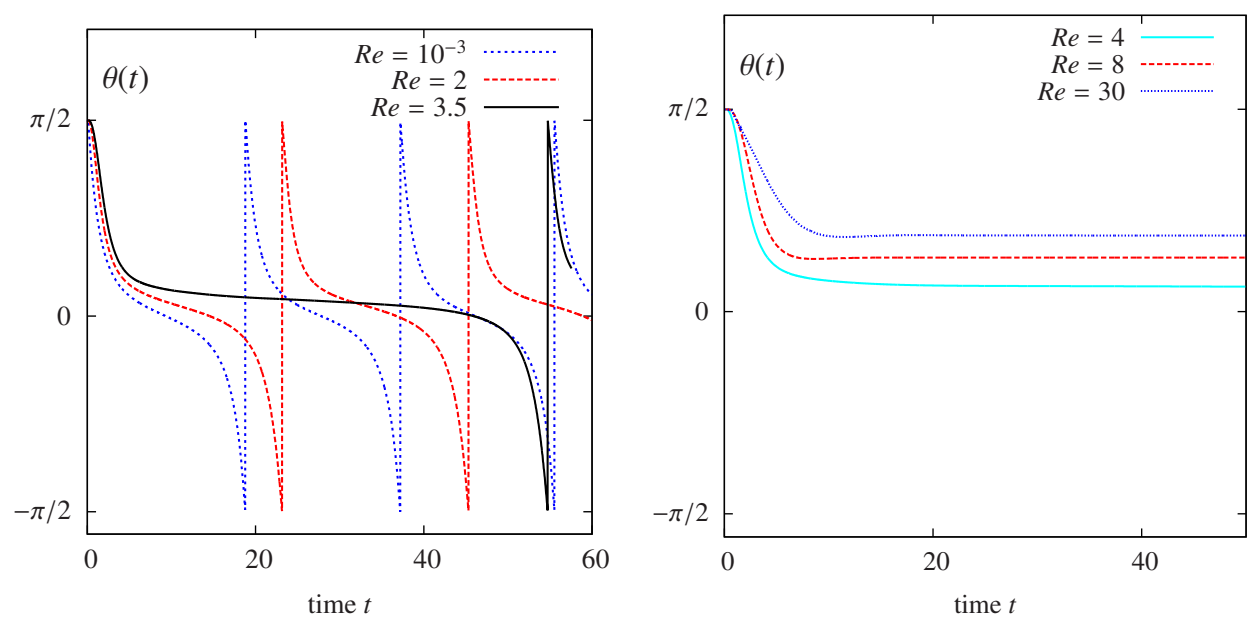

Figure 19: Inertia effect: influence of $R e$ on the vesicle inclination $\theta(t)$ for $C a=10^{4}, \alpha=1 / 2$, $\beta=10$ and $\gamma=0.82$. (a) tumbling regime when $R e \leq 3 / 5$; (b) tank-treading regime when $R e \geq 4$.

However, more developments, detailed analyzis of this phenomenon and discussions on the effect of the inertia on vesicles dynamics, such as phase diagram in the relevant parameter space, are provided in [35, 38]. Other relevant investigations of the dynamics of vesicles in flows, where both inertial and viscous effects are important, are founded in [50].

\section{Conclusion}

The new level method presented in this paper for the simulation of the vesicle dynamics exactly satisfies both the inextensibility membrane condition and the volume conservation : these properties are also true at the discrete level. We show that the proposed method, based on Lagrange multipliers, solves a lack of precision problem when dealing with the inextensibility constraints and the level set method. Moreover, an automatic adaptive method, used at each time step, enhance the prediction of the vesicle motion. With this procedure, we are able to accurately reproduce the change of regime, from tank-treading to tumbling, as observed when the viscosity ratio varies.

We exhibit the apperance of a new change of regime when the Reynolds number 

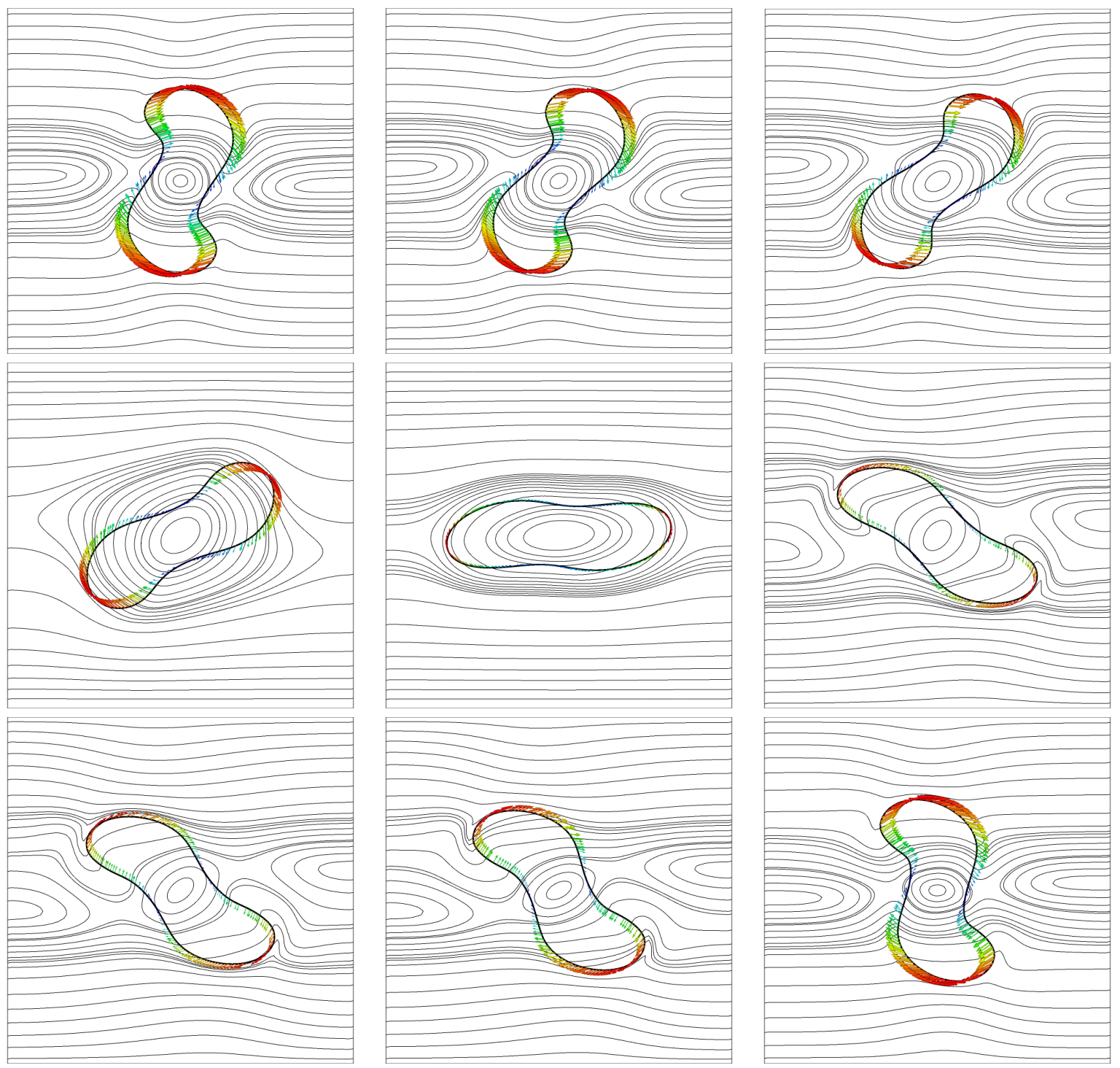

Figure 20: Inertia effects: streamlines lines and velocity field on the vesicle membrane for $R e=0.4, C a=10^{4}, \alpha=1 / 2, \beta=10$ and $\gamma=0.62$. Figure are shown, from left to right and top to bottom, at $t=k T_{p} / 24, k \in\{2,4,6,8,13,18,20,22,24\}$, where $T_{p}=29.1$ is the tumbling period.

is above a critical value. Moreover, the critical Reynolds number of this order of magnitude for both red blood cells in arteries and vesicles used in laboratory experiments. In the future, new experiments on vesicle would be necessary to infirm or confirm your numerical predictions. 
[1] R. A. Adams and J. J. F. Fournier. Sobolev spaces. Elsevier, second edition, 2003.

[2] S. M. Allen and J. W. Cahn. A microscopic theory for antiphase boundary motion and its application to antiphase domain coarsening. Acta Metal., 27:1085-1095, 1979.

[3] G. Aubert and P. Kornprobst. Mathematical problems in image processing. Partial differential equations and the calculus of variations. Springer, second edition, 2006.

[4] J.W. Barrett, H. Garcke, and R. Nürnberg. Parametric approximation of Willmore flow and related geometric evolution equations. Technical Report 22/2007, Univ. Regensburg, Deustchland, 2007.

[5] J. Beaucourt, F. Rioual, T. Seon, T. Biben, and C. Misbah. Steady to unsteady dynamics of a vesicle in a flow. Phys. Rev. E, 69:011906, 2004.

[6] T. Biben, K. Kassner, and C. Misbah. Phase-field approach to threedimensional vesicle dynamics. Phys. Rev. E, 72:041921, 2005.

[7] T. Biben and C. Misbah. Tumbling of vesicles under shear flow within an advected-field approach. Phys. Rev. E, 67:031908, 2003.

[8] G. Boedec, M. Leonetti, and M. Jaeger. 3d vesicle dynamics simulations with a linearly triangulated surface. J. Comput. Phys., 230(4):1020-1034, 2011.

[9] A. Bonito, R. H. Nochetto, and M. S. Pauletti. Parametric FEM for geometric biomembranes. J. Comput. Phys., 229(9):3171-3188, 2010.

[10] S. C. Brenner and L. R. Scott. The mathematical theory of finite element methods. Springer, third edition, 2008.

[11] F. Brezzi and M. Fortin. Mixed and hybrid finite element methods. Springer, 1991.

[12] C. Bui, V. Lleras, and O. Pantz. Dynamics of red blood cells in 2d. ESAIM Proc., 28:182-194, 2009.

[13] P. B. Canham. The minimum energy of bending as a possible explanation of the biconcave shape of the human red blood cell. J. Theoret. Biol., 26:61-81, 1970.

[14] U. Clarenz, U. Diewald, G. Dziuk, M. Rumpf, and R. Rusu. A finite element method for surface restoration with smooth boundary conditions. Computer Aided Geometric Design, 21(5):427-445, 2004.

[15] G.-H. Cottet, E. Maitre, and T. Milcent. Eulerian formulation and level-set models for incompressible fluid-structure interaction. Math. Model. Numer. Anal., 42:471-492, 2008. 
[16] V. Doyeux, V. Chabannes, C. Prud'Homme, and M. Ismail. Siimulation pf vesicle using level set method solved by high order finite element. ESAIM Proc., submited, 2012.

[17] Q. Du, C. Liu, R. Ryham, and X. Wang. Energetic variational approaches in modeling vesicle and fluid interactions. Physica D, 238:923-930, 2009.

[18] Q. Du, C. Liu, and X. Wang. A phase field approach in the numerical study of the elastic bending energy for vesicle membranes. J. Comput. Phys., 198:450-468, 2004.

[19] Q. Du and J. Zhang. Adaptive finite element method for a phase field bending elasticity model of vesicle membrane deformation. SIAM J. Ssci. Comput., 30(3):1634-1657, 2008.

[20] L. Formaggia and S. Perotto. New anisotropic a priori error estimates. Numer. Math., 89:641-667, 2001.

[21] M. Fortin and R. Glowinski. Augmented Lagrangian methods. Elsevier, 1983.

[22] V. Girault and P. A. Raviart. Finite element methods for the Navier-Stokes equations. Theory and algorithms. Springer, 1986.

[23] G. H. Golub and C. F. van Loan. Matrix computations. The John Hopkins University Press, Baltimore, MD, USA, third edition, 1996.

[24] F. Hecht. BAMG: bidimensional anisotropic mesh generator, 2006. http://www.ann.jussieu.fr/ hecht/ftp/bamg.

[25] W. Helfrich. Elastic properties of lipid bilayers: theory and possible experiments. Z. Naturforsch, 28(11):693-703, 1973.

[26] W. Huang. Metric tensors for anisotropic mesh generation. J. Comput. Phys., 204:633-665, 2005.

[27] W. Huang and X. Li. An anisotropic mesh adaptation method for the finite element solution of variational problems. Finite Elements in Analysis and Design, 46:61-73, 2010.

[28] S. Hysing. A new implicit surface tension implementation for interfacial flows. Int. J. Numer. Meth. Fluids, 51(6):659-672, 2006.

[29] D. Jamet and C. Misbah. Toward a thermodynamically consistent picture of the phase-field model of vesicles: curvature energy. Phys. Rev. E, 78:031902, 2008.

[30] V. Kantsler and V. Steinberg. Transition to tumbling and two regimes of tumbling motion of a vesicle in shear flow. Phys. Rev. Lett., 96:036001, Jan 2006. 
[31] S. Keller and R. Skalak. Motion of a tank-treading ellipsoidal particle in a shear flow. J. Fluid Mech., 120:27-47, 1982.

[32] Y. Kim and M.-C. Lai. Simulating the dynamics of inextensible vesicles by the penalty immersed boundary method. J. Comput. Phys., 229(12):48404853, 2010.

[33] M. Kraus, W. Wintz, U. Seifert, and R. Lipowsky. Fluid vesicles in shear flow. Phys. Rev. Lett., 77:3685-3688, Oct 1996.

[34] T. Krüger, F. Varnik, and D. Raabe. Efficient and accurate simulations of deformable particles immersed in a fluid using a combined immersed boundary lattice Boltzmann finite element method. Comput. Math. Appl., 61(12):3485-3505, 2011.

[35] A. Laadhari. Numerical modelling of the red blood cells dynamics using the level set method. Université De Grenoble, 2011. http://tel.archives-ouvertes.fr/tel-00598251.

[36] A. Laadhari, C. Misbah, and P. Saramito. On the equilibrium equation for a generalized biological membrane energy by using a shape optimization approach. Phys. D, 239:1568-1572, 2010.

[37] A. Laadhari, P. Saramito, and C. Misbah. Improving the mass conservation of the level set method. C. R. Acad. Sci. Paris, ser. I, 348:535-540, 2010.

[38] A. Laadhari, P. Saramito, and C. Misbah. Vesicle tumbling inhibited by inertia. Phys. Fluids, 24:031901, 2012.

[39] D.V. Le and S.T. Wong. A front-tracking method with catmull-clark subdivision surfaces for studying liquid capsules enclosed by thin shells in shear flow. Journal of Computational Physics, 230(9):3538-3555, 2011.

[40] L. Ma and W. S. Klug. Viscous regularization and r-adaptive remeshing for finite element analysis of lipid membrane mechanics. J. Comput. Phys., 227(11):5816-5835, 2008.

[41] E. Maitre, T. Milcent, G.-H. Cottet, A. Raoult, and Y. Usson. Applications of level set methods in computational biophysics. Math. Comput. Model., 49(11-12):2161-2169, 2009.

[42] S. Osher and J. A. Sethian. Front propaging with curvature-dependent speed: agorithms based on Hamilton-Jacobi formulations. J. Comput. Phys., 79(12), 1988.

[43] Z.-C. Ou-Yang and W. Helfrich. Bending energy of vesicle membranes: general expressions for the first, second and third variation of the shape energy and applications to spheres and cylinders. Phys. Rev. A, 39(10):5280-5288, 1989. 
[44] O. Pironneau. On the transport-diffusion algorithm and its applications to the Navier-Stokes equations. Numerische Mathematik, 38(3):309-332, 1982.

[45] C. Pozrikidis. Numerical simulation of the flow-induced deformation of red blood cells. Annals of Biomedical Engineering, 31:1194-1205, 2003.

[46] A. Rahimian, S. K. Veerapaneni, and G. Biros. Dynamic simulation of locally inextensible vesicles suspended in an arbitrary two-dimensional domain, a boundary integral method. J. Comput. Phys., 229(18):6466-6484, 2010 .

[47] R. Rusu. An algorithm for the elastic flow of surfaces. Interfaces and Free Boundaries, 7(3):229-239, 2005.

[48] S. A. Safran. Statistical thermodynamics of surfaces, interfaces and membranes. Frontier in physics, vol. 90. Addison-Wesley, Reading, USA, 1994.

[49] D. Salac and M. Miksis. A level set projection model of lipid vesicles in general flows. J. Comput. Phys., 230:8192-8215, 2011.

[50] D. Salac and M. J. Miksis. Reynolds number effects on lipid vesicles. Journal of Fluid Mechanics, 711:122-146, 112012.

[51] P. Saramito. Efficient C++ finite element computing with Rheolef. CNRS and LJK, 2011. http://cel.archives-ouvertes.fr/cel-00573970.

[52] U. Seifert. Configurations of fluid membranes and vesicles. Adv. Phys, 46:13-137, 1997.

[53] M. Sussman and E. Fatemi. An efficient, interface preserving level set redistancing algorithm and its application to interfacial incompressible fluid flow. SIAM J. Sci. Comput, 20(4):1165-1191, 1998.

[54] V. Vitkova, G. Coupier, M.-A. Mader, B. Kaoui, C. Misbah, and T. Podgorski. Tumbling of viscous vesicles in a linear shear field near a wall. Journal of optoelectronics and advanced materials, 11:1218-1221, 2009.

[55] P. M. Vlahovska, T. Podgorski, and C. Misbah. Vesicles and red blood cells in flow: From individual dynamics to rheology. C. R. Physique, 10:775-789, 2009 .

[56] T. Williams and C. Keley. gnuplot: an interactive program, 2010. http://www. gnuplot.info.

[57] H. Zhao and E. S. G. Shaqfeh. The dynamics of a vesicle in simple shear flow. Journal of Fluid Mechanics, 674:578-604, 2011. 


\section{AppendixA. Remark on the spontaneous curvature}

Let denote by $V_{0}$ the area and by $A_{0}$ the perimeter of the vesicle $\Omega$. Using as a characteristic length the radius $R_{0}$ of the circle having the same perimeter as $\partial \Omega$, the relation between the Lagrangian $\mathscr{L}$ and its dimensionless counterpart $\widetilde{\mathscr{L}}$ writes:

$\widetilde{\mathscr{L}}(\widetilde{\Omega} ; \widetilde{\lambda}, \widetilde{p})=\frac{2 R_{0}}{k_{c}} \mathscr{L}(\Omega ; \lambda, p)=\int_{\partial \widetilde{\Omega}} \widetilde{H}^{2} \mathrm{~d} \widetilde{s}+\widetilde{\lambda}\left(\int_{\partial \widetilde{\Omega}} \mathrm{d} \widetilde{s}-\widetilde{A}_{0}\right)+\widetilde{p}\left(\int_{\widetilde{\Omega}} \mathrm{d} \widetilde{x}-\widetilde{V}_{0}\right)$.

where $\tilde{\lambda}=\frac{2}{k_{c}} \lambda R_{0}^{2}$ and $\widetilde{p}=\frac{2}{k_{c}} p R_{0}^{3}$. denote the dimensionless Lagrange multipliers. Recall that the reduced area $\gamma=\frac{V_{0}}{\pi} \times\left(\frac{2 \pi}{A_{0}}\right)^{2}=\frac{V_{0}}{\pi R_{0}^{2}}$. Then, for the dimensionless problem, the volume and area express $\widetilde{V}_{0}=\frac{V_{0}}{R_{0}^{2}}=\pi \gamma$ and $\widetilde{A}_{0}=\frac{A_{0}}{R_{0}}=2 \pi$. As a consequence, the reduced area $\gamma$ is the unique dimensionless number of this problem, that characterizes the stationary shape of the vesicle: others parameters, such as $k_{c}$, has no effects.

Let us turn to the effect of the spontaneous curvature $H_{0} \geq 0$ : The Lagrangian writes:

$$
\mathscr{L}(\Omega ; \lambda, p)=\frac{k_{c}}{2} \int_{\partial \Omega}\left(H-H_{0}\right)^{2} \mathrm{~d} s+\lambda\left(\int_{\partial \Omega} \mathrm{d} s-A_{0}\right)+p\left(\int_{\Omega} \mathrm{d} x-V_{0}\right) .
$$

From $\left(H-H_{0}\right)^{2}=H^{2}-2 H H_{0}+H_{0}^{2}$, notice first that the last $H_{0}^{2}$ term is constant and thus, has no effects in the minimization problem. The only term that depend upon $H_{0}$ is the second one, involving $H_{0} \int_{\partial \Omega} H \mathrm{~d} s$. Using the general shape derivative analysis framework [36] with $f(H)=H$, we get, for any vector field $\mathbf{u}$ :

$$
\frac{\partial}{\partial \Omega}\left(\int_{\partial \Omega} H \mathrm{~d} s\right)(\Omega) \cdot(\mathbf{u})=\int_{\partial \Omega} 2 K \mathbf{u} \cdot \mathbf{n} \mathrm{d} s .
$$

where $K$ is the Gauss curvature of $\partial \Omega$. The gauss curvature $K$ is equal to zero for two dimensional problems. Then, the equilibrium shape of bidimensional vesicle depends only on the reduced area $\gamma$. As a matter of fact, the spontaneous curvature $H_{0}$ is only pertinent for three-dimensional problems.

\section{AppendixB. Remarks on the Lagrange multipliers}

Let us introduce the following space of admissible velocities:

$$
\mathbb{K}\left(\mathbf{u}_{b}\right)=\left\{\mathbf{v} \in \mathbb{V}\left(\mathbf{u}_{b}\right) ; \operatorname{div} \mathbf{v}=0 \text { in } \Lambda \text { and } \operatorname{div}_{s} \mathbf{v}=0 \text { on } \partial \Omega\right\}
$$


For any shape $\Omega$ and any admissible velocity field $\mathbf{u}$ defined in $\Lambda$, the energy of the system is defined by:

$$
J(\mathbf{u})=\int_{\Lambda} \eta^{*}|D(\mathbf{u})|^{2} \mathrm{~d} x+\frac{1}{2 C a} \int_{\partial \Omega} H^{2} \mathrm{~d} s,
$$

where |.| denotes the Euclidean norms of vectors or tensors. The previous expression of the energy includes two terms: the viscous energy involving $\mathbf{u}$ and the Canham-Helfrich bending energy, involving the shape $\Omega$ of the vesicle. Notice that, in the bending energy term in (B.1), the membrane $\partial \Omega$ depends upon the velocity field $\mathbf{u}$ via (3) and the level-set function $\phi$, satisfying the transport problem (4) that involves $\mathbf{u}$. Also, the curvature $H$ on $\partial \Omega$ depends implicitly upon $\mathbf{u}$. Remark that $J$ is not convex in general: the optimality system $J^{\prime}(\mathbf{u})=0$ could include both minimums and maximums of the energy $J$. Thus, this optimality system is not equivalent to the minimization of the energy. Nevertheless, a minimum of $J$ is also a solution of the optimality system.

An initial velocity field $\mathbf{u}_{0}$ is given. The problem is a strongly nonlinear shape optimization problem and writes:

$$
\mathbf{u}=\underset{\mathbf{v} \in \mathbb{K}\left(t, \mathbf{u}_{b}\right)}{\arg \inf } J(\mathbf{v}) .
$$

The space of admissible velocities $\mathbb{K}\left(t, \mathbf{u}_{b}\right)$ contains the incompressibility and inextensibility constraints: it is not suitable for practical finite element discretization, since there are no known finite element basis of such spaces. Conversely, the unconstrained space of $\mathbb{V}\left(\mathbf{u}_{b}\right)$ is of practical interest: the two constraints can be imposed via two Lagrange multipliers: the pressure $p$ and the surface tension $\lambda$. Let us introduce the following Lagrangian:

$$
\mathscr{L}(\mathbf{u} ; p, \lambda)=J(\mathbf{u})+\int_{\Lambda} p \operatorname{div} \mathbf{u} \mathrm{d} x+\int_{\partial \Omega} \lambda \operatorname{div}_{s} \mathbf{v} \mathrm{d} s .
$$

The previous minimization problem can be rewritten as a saddle point problem:

$$
(\mathbf{u}, p, \lambda)=\underset{\mathbf{v} \in \mathbb{V}\left(\mathbf{u}_{b}\right)}{\arg \inf } \sup _{\substack{q \in L^{2}(\Lambda) \\ \mu \in H^{\frac{1}{2}}(\partial \Omega)}} \mathscr{L}(\mathbf{v} ; q, \mu) .
$$

The usual [1] space of the trace of elements of $H^{1}(\Omega)$ on $\partial \Omega$ is denoted by $H^{\frac{1}{2}}(\partial \Omega)$. The Lagrange multipliers $p$ and $\lambda$ are associated to the constraints of local mass and area conservations. However, the minimization and saddlepoints principles are limited to the cases with zero Reynolds numbers, but they allows us to better understand the Lagrange multipliers significances and to perform suitable mass corrections in section 3. Moreover, section 3 shows that, after time discretization, a similar saddle-point problem has to be solved at each time step. 


\section{AppendixC. Remark on the redistanciation procedure}

Let us consider the transport equation: $D_{t} \phi=\partial_{t} \phi+\mathbf{u} \cdot \nabla \phi=0$. Using the summation of repeated indices convention, we get: $\partial_{i} \phi \partial_{i} \partial_{t} \phi+\partial_{i} \phi \partial_{i}\left(u_{j} \partial_{j} \phi\right)=0$ that writes also equivalently: $(1 / 2) \partial_{t}\left(|\nabla \phi|^{2}\right)+|\nabla \phi|^{2}(\mathbf{n} \otimes \mathbf{n}): \mathbf{u}+\partial_{i} \phi . \partial_{i}\left(u_{j} \partial_{j} \phi\right) . \quad$ Remark that: $\partial_{i} \phi . \partial_{i}\left(u_{j} \partial_{j} \phi\right)=(1 / 2) u_{j} \cdot \partial_{j}\left(\left(\partial_{i} \phi\right)^{2}\right)=(1 / 2) \mathbf{u} \cdot \nabla\left(|\nabla \phi|^{2}\right)=|\nabla \phi| \mathbf{u} \cdot \nabla(|\nabla \phi|)$.

Then, we obtain: $D_{t}(|\nabla \phi|)=|\nabla \phi|\left(\operatorname{div}_{s} \mathbf{u}-\operatorname{div} \mathbf{u}\right)$. The density of the fluid is supposed to be constant, and the mass conservation leads to $\operatorname{div} \mathbf{u}=0$. Moreover, in the context of vesicles, $\operatorname{div}_{s} \mathbf{u}=0$ since the membrane is supposed to be inextensible. Thus $D_{t}(|\nabla \phi|)=0$. When $|\nabla \phi|=1$ at $t=0$, i.e. when $\phi$ is initially a distance function, this property is then preserved for all $t>0$. When using the finite element approximation, we observe that this property is only approximately preserved, and thus, the redistancing procedure described in this paper is applied.

\section{AppendixD. Computation of the vesicle inclination}

This appendix presents the computation of the angle $\theta$ of the shape $\Omega$. Let $\left(x_{1}, x_{2}\right)$ be the coordinate system for $\mathbb{R}^{2}$, containing the shape $\Omega$ and $\mathrm{d} x=\mathrm{d} x_{1} \mathrm{~d} x_{2}$. The center of the vesicle is denoted by $\left(\bar{x}_{1}, \bar{x}_{2}\right)$, where $\bar{x}_{1}=\left(\int_{\Omega} x_{1} \mathrm{~d} x\right) / \operatorname{meas}(\Omega)$ and $\bar{x}_{2}=\left(\int_{\Omega} x_{2} \mathrm{~d} x\right) /$ meas $(\Omega)$. Let $I$ be the inertia matrix of the vesicle relative to the vertical axis in $\left(\bar{x}_{1}, \bar{x}_{2}\right)$ :

$$
I_{O}=\left(\begin{array}{cc}
\int_{\Omega}\left(x_{1}-\bar{x}_{1}\right)^{2} \mathrm{~d} x & \int_{\Omega}\left(x_{1}-\bar{x}_{1}\right)\left(x_{2}-\bar{x}_{1}\right) \mathrm{d} x \\
\int_{\Omega}\left(x_{1}-\bar{x}_{1}\right)\left(x_{2}-\bar{x}_{1}\right) \mathrm{d} x & \int_{\Omega}\left(x_{2}-\bar{x}_{1}\right)^{2} \mathrm{~d} x
\end{array}\right) .
$$

This symmetric matrix has two real eigenvalues and orthogonal eigenvectors. The inclination angle $\theta$ is defined as the angle between the eigenvector associated to the largest eigenvalue, and the $x_{1}$ axis. 